\title{
Compressed Sensing Image Reconstruction Via Adaptive Sparse Nonlocal Regularization
}

\author{
Zhiyuan Zha · Xin Liu • Xinggan \\ Zhang - Yang Chen • Lan Tang • \\ Yechao Bai · Qiong Wang · Zhenhong \\ Shang
}

Received: date / Accepted: date

\begin{abstract}
Compressed Sensing (CS) has been successfully utilized by many computer vision applications. However,the task of signal reconstruction is still challenging, especially when we only have the CS measurements of an image (CS image reconstruction). Compared with the task of traditional image restoration (e.g., image denosing, debluring and inpainting, etc.), CS image reconstruction has partly structure or local features. It is difficulty to build a dictionary for CS image reconstruction from itself. Few studies have shown promising reconstruction performance since most of existing methods employed a fixed set of bases (e.g., wavelets, DCT, and gradient spaces) as the dictionary, which lack the adaptivity to fit image local structures. In this paper, we propose an adaptive sparse nonlocal regularization (ASNR) approach for CS image reconstruction. In ASNR, an effective self-adaptive learning dictionary is used to greatly reduce artifacts and the loss of fine details. The dictionary is compact and learned from the reconstructed image itself rather than natural image dataset. Furthermore, the image sparse nonlocal (or nonlocal self-similarity) priors are integrated into the regularization term, thus ASNR can effectively enhance the quality of the CS image reconstruction. To

Z. Zha $\cdot$ X. Zhang $(\bowtie) \cdot$ Y. Chen · Y. Bai · Q. Wang zhxg@nju.edu.cn

School of Electronic Science and Engineering, Nanjing University, Nanjing 210023, China.

X. Liu

The Center for Machine Vision and Signal Analysis, University of Oulu, Finland. And School of Electronics and Information Engineering, Xi'an Jiaotong University, Xi'an, Shanxi, China.

L. Tang

Department of Electronic Science and Engineering, Nanjing University, and National Mobile Commun. Research Lab., Southeast University, Nanjing 210023, China.

Z. Shang

School of Information Engineering and Automation, Kunming University of Science and Technology, Kunming 650500, China.
\end{abstract}


improve the computational efficiency of the ASNR, the split Bregman iteration based technique is also developed, which can exhibit better convergence performance than iterative shrinkage/thresholding (IST) method. Extensive experimental results demonstrate that the proposed ASNR method can effectively reconstruct fine structures and suppress visual artifacts, outperforming state-of-the-art performance in terms of both the PSNR and visual measurements.

Keywords Compressed sensing · Nonlocal self-similarity · Dictionary learning $\cdot$ split Bregman iteration

\section{Introduction}

The recent development of the compressed sensing (CS) theory [1,2] has drawn much attention in signal/image communities. The CS theory has uncovered that by achieving the possibility of reconstructing a signal from a smaller number of random measurements, as long as the signal has a sparse representation in some domain. The CS theory has the potential of dramatically improving the energy efficiency of sensors, leading to extensive applications in the real world. For instance, in recent years, several CS-based imaging systems have been established, including the compressed spectral imaging system [3], the single-pixel camera [4], and the high-speed video camera [5].

In the CS theory, if a signal is sparse in some transform domain, it is usually sampled by a random projection which is independent and reconstructed by minimizing the $\ell_{0}$ norm or the $\ell_{1}$ norm optimization problem with some prior information which usually constitutes regularization terms. However, since the $\ell_{0}$ norm minimization is a difficult combinatorial optimization problem, solving this problem directly is both NP-hard and unstable in the presence of noise. For this reason, it has been proposed to replace the non-convex $\ell_{0}$ norm by its convex $\ell_{1}$ norm, and provably it is probable that the $\ell_{1}$ norm is equivalent to the $\ell_{0}$ norm in many practical problems. Therefore, under these circumstances, one is allowed to solve the problem easily by the $\ell_{1}$ norm, rather than the $\ell_{0}$ norm. The optimization problem by the $\ell_{1}$ norm can be solved by linear programming [6] and several CS restoration algorithms, known as the gradient projection sparse reconstruction [7], the orthogonal matching pursuit [8], and the iterative thresholding [9], which have been proposed recently.

As a basic image inverse problem in the field of image restoration, the purpose of CS image reconstruction is to reconstruct high quality image from considerably fewer measurements than suggested by the Nyquist sampling theory. In addressing this issue, the most effective method now is to utilize image prior knowledge or some constraints, which plays a key role in the CS image reconstruction problems to recover the missing information. Accordingly, effective regularization terms are designed to reflect the image priors, which is vital for the CS image reconstruction.

The classic regularization models, such as the quadratic Tikhonov regularization model [10], the Mumford-Shah (MS) model [11], and the TV regular- 
ization model [12], utilize the local structure patterns of an image and high effectiveness to preserve image edges and recover the smooth regions of an image. Nevertheless, they usually destroy the image details and cannot handle fine structures well, since they have only exploited the image local statistics, while ignoring the nonlocal statistics.

Besides the local structural schema, perhaps the most remarkable nonlocal statistics are the nonlocal self-similarity models demonstrated by natural images in the image processing, which was initially proposed as a solution to image denoising, called the non-local means (NLM) denoising filtering [13]. The basic idea of the nonlocal self-similarity method is very simple: the image patches that have similar patterns can be spatially far from each other and thus we can collect them from the whole image. The nonlocal self-similarity characterizes the repetitiveness of textures and structures reflected by natural images within nonlocal regions, which can be applied to retain the edges and the sharpness effectively to maintain image nonlocal consistency [13-16]. Later, inspired by the success of the NLM denoising filtering, a flurry of nonlocal regularization terms were proposed to solve the image inverse problems and to utilize the nonlocal self-similarity properties of natural images as exhibited in [14-19]. Note that the NLM-based regularization term in [17] is implemented at the pixel level, i.e., from one pixel to another pixel. The block-level NLM-based method is introduced in [14] to address the image deblurring and super-resolution problems. The connection between the spectral bases of the nonlocal graph Laplacian operator and the filtering methods was proposed by Peyr in [15]. Jung et al. [18] proposed a nonlocal form of the approximation of the MS regularizer for color image restoration instead of the traditional local MS regularizer. Recently, Dong et al. [19] proposed a nonlocally centralized sparse representation model for image restoration, which first obtains good estimates of the sparse coding coefficients of the original image, and then centralizes the sparse coding coefficients of the observed image to those estimates to improve the performance of sparse representation-based image restoration.

In recent years, the patch-based sparse representation along with dictionary learning (DL) from natural images, has led to very impressive reconstruction results $[14,19-23]$. The sparse representation model assumes that each patch of an image can be accurately represented by a few elements from a basis set called a dictionary, which is usually learned from a natural image dataset [20]. Therefore, one important issue of the sparsity-based image restoration is the selection of the dictionary. The conventional analytically designed dictionaries, such as the DCT, wavelet, bandlet and curvelet, etc., share the superiority of fast conduct, yet they usually lack the adaptivity to fit the image local structures, resulting in poor reconstruction performance. Compared with these predefined sparse transforms, the DL method eliminates the disadvantage of a fixed sparse transform. For example, it is impossible for one predefined sparse transform to be universally optimal and sufficient to characterize the many complex structures of natural images. The purpose of many DL methods is to learn a universal and over-complete dictionary which can represent various image structures. However, it has been shown that sparse decomposition 
over an over-complete dictionary is potentially unstable, and it tends to generate visual artifacts $[24,25]$. Recently, the PCA sub-dictionary has been an illustrious DL method, which clusters the training patches extracted from a set of natural images into some clusters, and finally learns a compact PCA sub-dictionary for each cluster [14].

To reconstruct an image accurately from its compressed sensing measurements, Wu et al. [26] proposed a model-assisted adaptive recovery of compressed sensing for exploiting the $2 \mathrm{D}$ piecewise autoregressive model and, in turn, for making the CS recovery adaptive to spatially transform the second order statistics of an image. Ravishankar et al. [27] began with a rough estimate for the compressed sensing measurement, and they simultaneously updated the sparse dictionary and the sparse coefficients of all overlapped patches, and finally averaged all the reconstructed patches to estimate the image iteratively. Many recent image CS reconstruction works integrated some prior knowledge into the image CS reconstruction framework, such as the tree-structured wavelet [28], tree-structured DCT [29] , and Gaussian mixture models [30] have integrated some prior knowledge into the CS image reconstruction framework. Mun et al. [31] proposed a projection-driven CS reconstruction incorporated with block-based random image sampling, which aimed at encouraging sparsity in the domain of directional transforms.

Different from the task of traditional image restoration (e.g, image denoising, deblurring, inpainting, etc.), which can directly use the image nonlocal self-similarity (NSS) property and due to the encouragement by the recen$\mathrm{t}$ excellent sparse representation methods in the image processing. In this paper, we propose a unified variational framework for adaptive sparse nonlocal regularization model of CS image reconstruction. Specifically, an effective self-adaptive dictionary learning as sparse domain is used to greatly reduce visual artifacts and the loss of fine details. The dictionary is compact and learned from the reconstructed image itself rather than natural image dataset. In addition, the image sparse nonlocal (or nonlocal self-similarity) priors are integrated into the regularization term to enhance the quality of CS image reconstruction. To improve the computational efficiency of the proposed method, we have developed a convenient implementation utilizing the split Bregman iteration technique, which can exhibit good convergence performance. Extensive experimental results validate that the proposed method can effectively reconstruct fine structures and suppress visual artifacts, outperforming many current state-of-the-art schemes in terms of both the PSNR and visual measurements.

The rest of this paper is organized as follows:-Section 2 briefly states the prior work in the CS theory and the patch-based sparse representation. Section 3 presents the modeling of the ASNR and introduces the implementation details. Section 4 presents the extensive experimental results, and Section 5 concludes this paper. 


\section{Background and related work}

\subsection{Compressed sensing}

The CS theory demonstrates that the signal can be recovered by linear projection from its measurements, which may even be far below the traditional Nyquist sampling rate, as long as the signal has a sparse representation in some domain. In the CS theory, a signal $\boldsymbol{x}$ of size $N$ is viewed as sparse in domain $\Psi$, if its transform $\theta$ coefficients over $\Psi$ is expressed by $\boldsymbol{x}=\Psi \theta$, where $\theta$ are mostly zeros or nearly sparse if the vast majority of coefficients $\theta$ are either zeros or very close to zero. The number of significant elements within the coefficient vector $\theta$ is usually quantifying the sparsity of $\boldsymbol{x}$ in $\Psi$.

More specifically, one can seek the perfect reconstruction of a signal $\boldsymbol{x}$ from $\boldsymbol{y} \in \Re^{\mathbf{M}}$ with $M$ randomized linear measurements, which is formulated as the following constrained optimization problem, namely,

$$
\arg \min _{\theta}\|\theta\|_{0}, \quad \text { s.t. } \quad \boldsymbol{y}=\boldsymbol{\Phi} \Psi \theta
$$

where $\boldsymbol{\Phi} \in \Re^{\mathbf{M} \times \mathbf{N}}$ represents the random projection matrix and such that $M$ is much smaller than $N$. $\|\bullet\|_{0}$ represents the $\ell_{0}$ norm, counting the number of nonzero entries of a vector $\theta$.

However, since the $\|\bullet\|_{0}$ norm minimization is discontinuous and an NPhard problem, it is often relaxed to the convex $\ell_{1}$ norm minimization. By selecting an appropriate regularization parameter, Eq.(1) is equivalent to the following unconstrained optimization problem, -i.e.,

$$
\arg \min \frac{1}{2}\|\boldsymbol{y}-\boldsymbol{\Phi} \Psi \theta\|_{2}^{2}+\lambda\|\theta\|_{1}
$$

According to [32], CS is capable of recovering $K$-sparse signal $\boldsymbol{x}$ with a high probability from $M=O(K \log (N / K))$ random measurements by solving this $\ell_{1}$ norm optimization problem.

\subsection{Patch-based sparse representation}

Recently, the sparse representation based modeling has been proved to be a promising model for the image restoration studies [14,19-23], which assumes that a natural image is sparse in some domain spanned by a set of bases or the atoms of dictionary. According to [21], the basic unit of sparse representation for images is patch. Mathematically, for an image $\boldsymbol{x} \in \Re^{N}$, let $\boldsymbol{x}_{i}=\boldsymbol{R}_{i} \boldsymbol{x}$, $i=1,2, \ldots, n$ denote an image patch of size $\sqrt{b c} \times \sqrt{b c}$ extracted at location $i$, where $\boldsymbol{R}_{i}$ is the matrix extracting patch $\boldsymbol{x}_{i}$ from $\boldsymbol{x}$ at location $i$. Given a dictionary $\boldsymbol{D} \in \Re^{b c \times M}, b c \leq M$, the sparse coding processing of each patch $\boldsymbol{x}_{i}$ is to find a sparse vector $\boldsymbol{\alpha}_{i}$ to satisfy $\boldsymbol{x}_{i}=\boldsymbol{D} \boldsymbol{\alpha}_{i}$ over dictionary $\boldsymbol{D}$, where $\boldsymbol{\alpha}_{i}$ is a sparse vector whose coefficients are mostly zero or close to zero. Obviously, the image patches can be overlapped to better suppress noise and visual artifacts, and we can obtain a redundant patch-based representation. Then the entire 
image $\boldsymbol{x}$ can be reconstructed by averaging all the reconstructed patches $\boldsymbol{x}_{i}$, which can be mathematically expressed as

$$
\boldsymbol{x} \approx \boldsymbol{D} \boldsymbol{\alpha}=\left(\sum_{i=1}^{n} \boldsymbol{R}_{i}^{T} \boldsymbol{R}_{i}\right)^{-1}\left(\sum_{i=1}^{n} \boldsymbol{R}_{i}^{T} \boldsymbol{D} \boldsymbol{\alpha}_{i}\right)
$$

where $\boldsymbol{\alpha}$ denotes the concatenation of all $\boldsymbol{\alpha}_{i}$, that is, $\boldsymbol{\alpha}=\left[\boldsymbol{\alpha}_{1}^{T}, \boldsymbol{\alpha}_{2}^{T}, \ldots, \boldsymbol{\alpha}_{n}^{T}\right]^{T}$. Note that the above equation is nothing but signifying that the overall image is reconstructed by averaging each reconstructed patch of $\boldsymbol{x}_{i}$.

After this, we merged Eq.(3) into Eq.(1). The CS recovery scheme for sparse coding by the $\ell_{0}$ norm is formulated as

$$
\arg \min _{\boldsymbol{\alpha}}\|\boldsymbol{\alpha}\|_{0}, \quad \text { s.t. }, \quad \boldsymbol{y}=\boldsymbol{\Phi} \boldsymbol{D} \boldsymbol{\alpha}
$$

where $\boldsymbol{D}$ replaces $\Psi$ in Eq.(1), standing for a learning dictionary, and $\boldsymbol{\alpha}$ represents the patch-based sparse coding coefficients for the entire image over dictionary $\boldsymbol{D}$.

However, since the $\ell_{0}$ norm minimization is discontinuous and NP-hard, solving Eq.(4) is a difficult combinatorial optimization problem. On this account, a straightforward method has been proposed for solving the non-convex $\ell_{0}$ norm by its convex $\ell_{1}$ counterpart, and Eq.(4) can be turned into the following unconstrained optimization problem:

$$
\arg \min _{\boldsymbol{\alpha}} \frac{1}{2}\|\boldsymbol{y}-\boldsymbol{\Phi} \boldsymbol{D} \boldsymbol{\alpha}\|_{2}^{2}+\lambda\|\boldsymbol{\alpha}\|_{1}
$$

The above $\ell_{1}$-minimization problem is a classic sparse coding problem and can be efficiently solved by various methods, such as the iterative shrinkage algorithm [9], the split Bregman algorithm [33,34], and the alternative direction multiplier method [35].

\section{Modeling of compressed sensing image reconstruction via adaptive sparse nonlocal regularization}

Now, the goal is to reconstruct the desired image $\boldsymbol{x}$ from CS measurements $\boldsymbol{y}$. In the scenario of CS image reconstruction, this problem can be converted to decoding the CS measurements $\boldsymbol{y}$ so as to obtain the desired $\boldsymbol{\alpha}$

$$
\arg \min _{\boldsymbol{\alpha}} \frac{1}{2}\|\boldsymbol{y}-\boldsymbol{\Phi} \boldsymbol{D} \boldsymbol{\alpha}\|_{2}^{2}+\lambda \boldsymbol{R}(\boldsymbol{\alpha})
$$

where $\boldsymbol{R}(\boldsymbol{\alpha})$ represents some regularization terms and $\lambda$ represents the regularization parameter. However, in this paper, due to not knowing whether $\boldsymbol{R}(\boldsymbol{\alpha})$ is a convex or a non-convex minimization, we have developed a convenient implementation using the split Bregman iteration algorithm to solve Eq.(6) without considering the property of $\boldsymbol{R}(\boldsymbol{\alpha})$ in advance. 
Table 1: Split Bregman Iteration

\begin{tabular}{ll}
\hline & \multicolumn{1}{c}{ Split Bregman Iteration (SBI) } \\
\hline 1. & set $t=0$, choose $\mu>0, \boldsymbol{b}_{0}=0, \boldsymbol{z}_{0}=0, \boldsymbol{\alpha}_{0}=0$. \\
2. & Repeat \\
3. & $\boldsymbol{z}^{(t+1)}=\arg \min _{\boldsymbol{z}}(\boldsymbol{z})+\frac{\mu}{2}\left\|\boldsymbol{z}-\boldsymbol{G} \boldsymbol{\alpha}^{(t)}-\boldsymbol{b}^{(t)}\right\|_{2}^{2}$ \\
4. & $\boldsymbol{\alpha}^{(t+1)}=\arg \min _{\boldsymbol{\alpha}}(\boldsymbol{\alpha})+\frac{\mu}{2}\left\|\boldsymbol{z}^{(t+1)}-\boldsymbol{G} \boldsymbol{\alpha}-\boldsymbol{b}^{(t)}\right\|_{2}^{2}$ \\
5. & $\boldsymbol{b}^{(t+1)}=\boldsymbol{b}^{(t)}-\left(\boldsymbol{z}^{(t+1)}-\boldsymbol{G} \boldsymbol{\alpha}^{(t+1)}\right)$ \\
6. & $t \leftarrow t+1 ;$ \\
7. & Until $\quad$ stoping criterion is satisfied. \\
\hline
\end{tabular}

3.1 Split Bregman based iterative algorithm for the proposed ASNR model

In this section, we exploit the algorithmic framework of the split Bregman iteration $[33,34]$ to solve Eq.(6) and introduce the implementation details of the proposed method.

The split Bregman iteration (SBI) has recently been proposed by Goldstein [33] for solving a very broad class of $\ell_{1}$ norm related minimization problems. By exploiting the variable splitting technique, the SBI translates the unconstrained minimization problem into a constrained one, after which the constrained minimization problem can be solved by invoking the Bregman iteration $[33,34]$. Next, we will briefly introduce the SBI, by considering a constrained optimization problem, i.e. ,

$$
\min _{\boldsymbol{z} \in \Re^{\mathbf{N}}, \boldsymbol{\alpha} \in \Re^{\mathrm{M}}} f(\boldsymbol{z})+g(\boldsymbol{\alpha}), \quad \text { s.t. } \quad \boldsymbol{z}=\boldsymbol{G} \boldsymbol{\alpha}
$$

where $\boldsymbol{G} \in \Re^{\mathbf{M} \times \mathbf{N}}$ and $f: \Re^{\mathbf{N}} \rightarrow \Re, g: \Re^{\mathbf{M}} \rightarrow \Re$ are convex functions. Note that although $g(\boldsymbol{\alpha})$ is a convex function, it does not imply that it is infeasible when $g(\boldsymbol{\alpha})$ is a non-convex function, which has been done in [36]. The split Bregman iteration algorithm is described in Table 1.

In the SBI, the problem sequence approaching infinity can be avoided with the fixed parameter $\mu$, which has been done in [37]. According to the SBI, each sub-problem minimization may be simpler than solving the original problem directly if the original minimization is split into two sub-problems.

Now, let us come back to Eq.(6) and show how to exploit the framework of the SBI to solve it. We first convert Eq.(6) into another equivalent constrained form by introducing a variable $\boldsymbol{z}$, namely,

$$
\arg \min _{\boldsymbol{z}, \boldsymbol{\alpha}} \frac{1}{2}\|\boldsymbol{y}-\boldsymbol{\Phi} \boldsymbol{z}\|_{2}^{2}+\lambda \boldsymbol{R}(\boldsymbol{\alpha}), \quad \text { s.t. } \quad \boldsymbol{z}=\boldsymbol{D} \boldsymbol{\alpha}
$$

By defining $f(\boldsymbol{z})=\|\boldsymbol{y}-\boldsymbol{\Phi} \boldsymbol{z}\|_{2}^{2}, g(\boldsymbol{\alpha})=\lambda \boldsymbol{R}(\boldsymbol{\alpha})$, we have

$$
\boldsymbol{z}^{(t+1)}=\arg \min _{\boldsymbol{z}} \frac{1}{2}\|\boldsymbol{y}-\boldsymbol{\Phi} \boldsymbol{z}\|_{2}^{2}+\frac{\mu}{2}\left\|\boldsymbol{z}-\boldsymbol{D} \boldsymbol{\alpha}^{(t)}-\boldsymbol{b}^{(t)}\right\|_{2}^{2}
$$

and

$$
\boldsymbol{\alpha}^{(t+1)}=\arg \min _{\boldsymbol{\alpha}} \lambda \boldsymbol{R}(\boldsymbol{\alpha})+\frac{\mu}{2}\left\|\boldsymbol{z}^{(t+1)}-\boldsymbol{D} \boldsymbol{\alpha}-\boldsymbol{b}^{(t)}\right\|_{2}^{2}
$$


We can then update $\boldsymbol{b}^{(t)}$ by line 5 in Table 1 :

$$
\boldsymbol{b}^{(t+1)}=\boldsymbol{b}^{(t)}-\left(\boldsymbol{z}^{(t+1)}-\boldsymbol{D} \boldsymbol{\alpha}^{(t+1)}\right)
$$

Therefore, according to the SBI, the minimization for Eq.(6) is equivalent to solve two sub-problems, that is, $\boldsymbol{z}$ and $\boldsymbol{\alpha}$ sub-problems. Next, we will expound that every split sub-problem is suitable for an efficient solution. To avoid confusion, the subscribe $t$ may be omitted for conciseness.

1) $z$ sub-problem

Given $\boldsymbol{\alpha}$, the $\boldsymbol{z}$ sub-problem denoted by Eq.(9) becomes

$$
\arg \min _{z} \boldsymbol{Q}_{1}(\boldsymbol{z})=\arg \min _{\boldsymbol{z}} \frac{1}{2}\|\boldsymbol{y}-\boldsymbol{\Phi} \boldsymbol{z}\|_{2}^{2}+\frac{\mu}{2}\|\boldsymbol{z}-\boldsymbol{D} \boldsymbol{\alpha}-\boldsymbol{b}\|_{2}^{2}
$$

Clearly, $\boldsymbol{z}$ is actually a closed form of Eq.(12); $\boldsymbol{Q}_{1}(\boldsymbol{z})$ is a strictly convex quadratic function and its gradient can be expressed as

$$
\boldsymbol{d}\left(\boldsymbol{Q}_{1}(\boldsymbol{z})\right)=\boldsymbol{\Phi}^{T} \boldsymbol{\Phi} \boldsymbol{z}-\boldsymbol{\Phi}^{T} \boldsymbol{y}+\mu(\boldsymbol{z}-\boldsymbol{D} \boldsymbol{\alpha}-\boldsymbol{b})
$$

Setting $\boldsymbol{d}\left(\boldsymbol{Q}_{1}(\boldsymbol{z})\right)$ to be zero gives the exact minimization of Eq.(12), namely

$$
\hat{z}=\left(\boldsymbol{\Phi}^{T} \boldsymbol{\Phi}+\mu \boldsymbol{I}\right)^{-1}\left(\boldsymbol{\Phi}^{T} \boldsymbol{y}+\mu(\boldsymbol{D} \boldsymbol{\alpha}+\boldsymbol{b})\right)
$$

where $\boldsymbol{I}$ represents the identity matrix.

In the CS image reconstruction, since $\boldsymbol{\Phi}$ is a random projection matrix without a special structure, computing the inverse by Eq.(14) at each iteration is too costly to implement numerically. Therefore, to avoid computing the matrix inverse, an iterative method is highly desirable for solving Eq.(12). In this paper, we exploit the gradient descent method with the optimal step to solve Eq.(12), namely

$$
\hat{z}=z-\eta d
$$

where $\boldsymbol{d}$ is the gradient direction of the objective function $\boldsymbol{Q}_{1}(\boldsymbol{z}), \eta=\operatorname{abs}\left(\boldsymbol{d}^{T} \boldsymbol{d} / \boldsymbol{d}^{T} \boldsymbol{\partial} \boldsymbol{d}\right)$ is the optimal step, $\boldsymbol{\partial}=\boldsymbol{\Phi}^{T} \boldsymbol{\Phi}+\mu \boldsymbol{I}$, and $\boldsymbol{I}$ is the identity matrix.

Finally, it only requires an iterative calculation of the following equation to solve the $\boldsymbol{z}$ sub-problem, namely,

$$
\hat{z}=\boldsymbol{z}-\eta\left(\boldsymbol{\Phi}^{T} \boldsymbol{\Phi} \boldsymbol{z}-\boldsymbol{\Phi}^{T} \boldsymbol{y}+\mu(\boldsymbol{z}-\boldsymbol{D} \boldsymbol{\alpha}-\boldsymbol{b})\right)
$$

where $\boldsymbol{\Phi}^{T} \boldsymbol{\Phi}$ and $\boldsymbol{\Phi}^{T} \boldsymbol{y}$ can be calculated in advance, making the solving of the above equation more efficient.

2) $\boldsymbol{\alpha}$ sub-problem

Given $\boldsymbol{z}$, similarly, according to Eq.(10), the $\boldsymbol{\alpha}$ sub-problem can be written as

$$
\arg \min _{\boldsymbol{\alpha}} \boldsymbol{Q}_{2}(\boldsymbol{\alpha})=\arg \min _{\boldsymbol{\alpha}} \frac{1}{2}\|\boldsymbol{D} \boldsymbol{\alpha}-\boldsymbol{r}\|_{2}^{2}+\frac{\lambda}{\mu} \boldsymbol{R}(\boldsymbol{\alpha})
$$

where $\boldsymbol{r}=\boldsymbol{z}-\boldsymbol{b}$. 
To make Eq.(17) as tractable as possible, we will make a conversion: let $\boldsymbol{x}=\boldsymbol{D} \boldsymbol{\alpha}$, and then Eq.(17) can be rewritten as:

$$
\arg \min _{\boldsymbol{\alpha}} \boldsymbol{Q}_{2}(\boldsymbol{\alpha})=\arg \min _{\boldsymbol{\alpha}} \frac{1}{2}\|\boldsymbol{x}-\boldsymbol{r}\|_{2}^{2}+\frac{\lambda}{\mu} \boldsymbol{R}(\boldsymbol{\alpha})
$$

Note that it is difficult to solve Eq.(18) due to the unknown structure of $\boldsymbol{R}(\boldsymbol{\alpha})$. In this paper, a general assumption is made, with which even a closed form can be achieved. Specifically, $\boldsymbol{r}$ can be regarded as some type of noisy observation of $\boldsymbol{x}$, and then the assumption that each element of $\boldsymbol{e}=\boldsymbol{x}-\boldsymbol{r}$ follows an independent zero-mean distribution with variance $\sigma^{2}$ is made. The universality of the above assumption merits attention, which means that it needs to be, for example, neither Gaussian nor Laplacian nor a generalized Gaussian distribution (GGD) [38] process, but something more general. The following conclusion can be proved with this assumption.

Theorem 1: Defining $\boldsymbol{x}, \boldsymbol{r} \in \Re^{N}, \boldsymbol{x}_{i}, \boldsymbol{r}_{i} \in \Re^{n}, \boldsymbol{e}(j)$ is every element of the error vector $\boldsymbol{e}$, where $\boldsymbol{e}=\boldsymbol{x}-\boldsymbol{r}, j=1, \ldots, N$. Assume that $\boldsymbol{e}(j)$ follows an independent zero mean distribution with variance $\sigma^{2}$. Therefore, for any $\varepsilon>0$, we can represent the relationship between $\frac{1}{N}\|\boldsymbol{x}-\boldsymbol{r}\|_{2}^{2}$ and $\frac{1}{K} \sum_{i=1}^{n}\left\|\boldsymbol{x}_{i}-\boldsymbol{r}_{i}\right\|_{2}^{2}$ by the following property, namely,

$$
\lim _{\substack{N \rightarrow \infty \\ K \rightarrow \infty}} \boldsymbol{P}\left\{\left|\frac{1}{N}\right|\left|\boldsymbol{x}-\boldsymbol{r}\left\|_{2}^{2}-\frac{1}{K} \sum_{i=1}^{n}|| \boldsymbol{x}_{i}-\boldsymbol{r}_{i}\right\|_{2}^{2}\right|<\varepsilon\right\}=1
$$

where $\boldsymbol{P}(\bullet)$ represents the probability and $K=n \times k$. The detailed proof of the Theorem $\mathbf{1}$ is given in the APPENDIX A.

On the basis of Theorem 1, we have the following equation with very large probability (restricted to 1 ) at each iteration $t$, namely,

$$
\frac{1}{N}\|\boldsymbol{x}-\boldsymbol{r}\|_{2}^{2}=\frac{1}{K} \sum_{i=1}^{n}\left\|\boldsymbol{x}_{i}-\boldsymbol{r}_{i}\right\|_{2}^{2}
$$

Next, the following equation can be achieved by merging Eq.(20) into Eq.(18), that is

$$
\begin{aligned}
& \arg \min _{\boldsymbol{\alpha}} \frac{1}{2}\|\boldsymbol{x}-\boldsymbol{r}\|_{2}^{2}+\frac{\lambda}{\mu} \boldsymbol{R}(\boldsymbol{\alpha}) \\
& =\arg \min _{\boldsymbol{\alpha}} \frac{1}{2} \sum_{i=1}^{n}\left\|\boldsymbol{x}_{i}-\boldsymbol{r}_{i}\right\|_{2}^{2}+\frac{\lambda K}{\mu N} \sum_{i=1}^{n} \boldsymbol{R}_{i}(\boldsymbol{\alpha}) \\
& =\arg \min _{\boldsymbol{\alpha}} \frac{1}{2} \sum_{i=1}^{n}\left\|\boldsymbol{x}_{i}-\boldsymbol{r}_{i}\right\|_{2}^{2}+\tau \sum_{i=1}^{n} \boldsymbol{R}_{i}(\boldsymbol{\alpha})
\end{aligned}
$$

where $\tau=\frac{\lambda K}{\mu N}$. 
It is obvious that the minimization of Eq.(21) can be efficient by solving $n$ sub-problem for all the image patches $\boldsymbol{x}_{i}$. Each patch-based sub-problem can be calculated by:

$$
\begin{aligned}
\boldsymbol{\alpha}_{i} & =\arg \min _{\boldsymbol{\alpha}} \frac{1}{2} \sum_{i=1}^{n}\left\|\boldsymbol{x}_{i}-\boldsymbol{r}_{i}\right\|_{2}^{2}+\tau \sum_{i=1}^{n} \boldsymbol{R}_{i}(\boldsymbol{\alpha}) \\
& =\arg \min _{\boldsymbol{\alpha}} \frac{1}{2} \sum_{i=1}^{n}\left\|\boldsymbol{D} \boldsymbol{\alpha}_{i}-\boldsymbol{r}_{i}\right\|_{2}^{2}+\tau \sum_{i=1}^{n} \boldsymbol{R}_{i}(\boldsymbol{\alpha})
\end{aligned}
$$

Clearly, Eq.(22) can also be viewed as a sparse coding problem. Now, the question how to determine the specific structure of $\boldsymbol{R}(\boldsymbol{\alpha})$ is quite essential. It has been widely recognized that image prior knowledge plays a significant role in the performance of CS image reconstruction, and thus designing an effective regularization term to reflect image priors is at the core of CS image reconstruction. Therefore, several regularization terms have been developed in recent years. For instance, when $\boldsymbol{R}_{i}(\boldsymbol{\alpha})$ is $\left\|\boldsymbol{\alpha}_{i}\right\|_{1}$, Eq.(22) becomes a convex $\ell_{1}$ norm minimization problem and can be efficiently solved by iterative shrinkage algorithm [9]. Meanwhile, when $\boldsymbol{R}_{i}(\boldsymbol{\alpha})$ is $\left\|\boldsymbol{\alpha}_{i}\right\|_{0}$, Eq.(22) becomes a non-convex $\ell_{0}$ norm minimization problem, and apparently its constrained optimization form can be efficiently solved by the orthogonal matching pursuit (OMP) algorithm [20].

From the above analysis, it is clear to see that the SBI method solves not only the convex optimization, but also the non-convex optimization problem. However, since $\boldsymbol{r}$ is regarded as a good approximation of the original image $\boldsymbol{x}$ at each iteration, it is challenging to recover the true sparse coding coefficients $\boldsymbol{\alpha}$ from $\boldsymbol{r}$. Only using the local sparsity constraint $\left\|\boldsymbol{\alpha}_{i}\right\|_{1}$ or $\left\|\boldsymbol{\alpha}_{i}\right\|_{0}$ in Eq.(22) may not lead to a sufficiently accurate CS image reconstruction, and even $\left\|\boldsymbol{\alpha}_{i}\right\|_{0}$ is a non-convex $\ell_{0}$ norm minimization. Therefore, good priors of natural images are crucial to improve the CS image reconstruction performance. It is well-known that the image sparse coding coefficients $\boldsymbol{\alpha}$ are not randomly distributed due to the local and nonlocal correlations existing in natural images. In order to achieve a sufficiently accurate CS reconstruction performance, we first use the NCSR model [19] as a regularization term instead of a conventional local sparsity constraint term for the CS image reconstruction, that is,

$$
\Sigma_{i}\left\|\boldsymbol{\alpha}_{i}-\boldsymbol{u}_{i}\right\|_{1}
$$

where $\boldsymbol{u}_{i}$ represent some reasonably good approximation of the sparse-coded patch-based original image $\boldsymbol{x}$.

In our CS reconstruction method, although the original image $\boldsymbol{x}$ is unknown, for each patch $\boldsymbol{x}_{i}$ in image $\boldsymbol{r}$, we search the nonlocal similar patches for it from within a large enough window in image $\boldsymbol{r}$, because $\boldsymbol{r}$ can be regarded as a good approximation of the original image $\boldsymbol{x}$. We exploit the weighted average of the first $\boldsymbol{x}_{i}^{q}$ patches closest to $L$ to predict $\boldsymbol{x}_{i}^{\prime}$, that is, $\boldsymbol{x}_{i}^{\prime}=\Sigma_{q=1}^{L} b_{i}^{q} \boldsymbol{x}_{i}^{q}$. The weight $b_{i}^{q}$ is set to be inversely proportional to the distance between patches $\boldsymbol{x}_{i}$ and $\boldsymbol{x}_{i}^{q}: b_{i}^{q}=\exp \left(-\left\|\boldsymbol{x}_{i}-\boldsymbol{x}_{i}^{q}\right\|_{2}^{2} / h\right) / W$, where $h$ is a predetermined scalar and 
$W$ is the normalization factor. In the case of an orthogonal dictionary (e.g., the local PCA dictionary used in this work, see sub-section 3.2 for more details), each patch $\boldsymbol{x}_{i}$ of $\boldsymbol{r}$ and its nonlocal prediction patch $\boldsymbol{x}_{i}^{\prime}$ are easily coded by a dictionary $\boldsymbol{D}_{i}$, that is, $\boldsymbol{x}_{i}=\boldsymbol{D}_{i} \boldsymbol{\alpha}_{i}$ and $\boldsymbol{x}_{i}^{\prime}=\boldsymbol{D}_{i} \boldsymbol{u}_{i}$. Since the dictionary is orthogonal, the sparse coding coefficients $\boldsymbol{\alpha}_{i}$ and $\boldsymbol{u}_{i}$ should be similar, and,

$$
\boldsymbol{u}_{i}=\boldsymbol{D}_{i}^{-1} \boldsymbol{x}_{i}^{\prime}
$$

Accordingly, we can exploit $\boldsymbol{R}_{i}(\boldsymbol{\alpha})=\Sigma_{i}\left\|\boldsymbol{\alpha}_{i}-\boldsymbol{u}_{i}\right\|_{p}$ to regularize the solution of Eq.(22), namely,

$$
\boldsymbol{\alpha}_{i}=\arg \min _{\boldsymbol{\alpha}_{i}} \frac{1}{2} \sum_{i=1}^{n}\left\|\boldsymbol{D} \boldsymbol{\alpha}_{i}-\boldsymbol{r}_{i}\right\|_{2}^{2}+\tau \Sigma_{i}\left\|\boldsymbol{\alpha}_{i}-\boldsymbol{u}_{i}\right\|_{p}
$$

where $p$ denotes the $\ell_{p}$ norm. In general, $p$ is equal to 1 , which leads to the Laplacian distribution and $p$ is equal to 2, which follows the Gaussian distribution. If $0<p<1$, which follows the hyper-Laplacian distribution.

Let

$$
\chi_{i}=\boldsymbol{\alpha}_{i}-\boldsymbol{u}_{i}
$$

Here we perform some experiments to investigate the statistical property of $\chi$, where $\chi$ represents the set of $\chi_{i}$. We have tested the fidelity term in Eq.(25) on the image Leaves with $0.2 \mathrm{~N}$ measurements, Monarch with $0.3 \mathrm{~N}$ measurements and boats with $0.4 N$ measurements, respectively. We plot the histogram of $\chi$ as well as the fitting Gaussian, Laplacian and hyper-Laplacian distributions of $\chi$ in Fig.1(a), Fig.2(a) and Fig3.(a), respectively. To better observe the fitting of the tails, we also plot these distributions in the log domain in Fig.1(b), Fig.2(b) and Fig.3(b), respectively. It can be observed that the histogram of $\chi$ can be characterized well by the Laplacian distribution. Therefore, the $\ell_{1}$ norm is adopted to regularize $\chi$, and the proposed model can be formulated as:

$$
\boldsymbol{\alpha}_{i}=\arg \min _{\boldsymbol{\alpha}_{i}} \frac{1}{2} \sum_{i=1}^{n}\left\|\boldsymbol{D} \boldsymbol{\alpha}_{i}-\boldsymbol{r}_{i}\right\|_{2}^{2}+\tau \Sigma_{i}\left\|\boldsymbol{\alpha}_{i}-\boldsymbol{u}_{i}\right\|_{1}
$$

Note that for fixed $\tau, \boldsymbol{u}_{i}$, Eq.(27) is convex and can be achieved efficiently by using an iterative thresholding algorithm. We adopt the surrogate algorithm in [9] to solve it. In the $(t+1)$-iteration, the proposed shrinkage operator can be calculated as

$$
\boldsymbol{\alpha}_{i}^{(t+1)}=S_{\rho}\left(\boldsymbol{D}^{-1} \boldsymbol{x}_{i}^{(t)}-\boldsymbol{u}_{i}^{(t)}\right)+\boldsymbol{u}_{i}^{(t)}
$$

where $S_{\rho}(\bullet)$ is the soft thresholding operator, $\rho=\tau / c$ and $c$ is an auxiliary parameter. The above shrinkage operator follows the standard surrogate algorithm, from which more details can be seen in [9]. Hence, if we know the dictionary $\boldsymbol{D}, \boldsymbol{\alpha}$ can be obtained by sparse coding all the overlapped patches, which is the final solution for $\boldsymbol{\alpha}$ sub-problem in Eq.(17). In the next subsection, we will discuss how to learn a self-adaptive dictionary $\boldsymbol{D}$.

According to the above analysis, the main difference between [19] and this paper for sparse coding coefficient $\boldsymbol{\alpha}$ is that we utilize the spit Bregman 
iteration method to solve a sub-problem to achieve $\boldsymbol{\alpha}$, while [19] solves $\boldsymbol{\alpha}$ by a direct method. The rationale behind is that each sub-problem minimization may be more effective and robust than the original problem. In addition, the iterative shrinkage (IS) algorithm [9] is applied to solve the original problem in [19]. However, the major drawback of the IS algorithm is its low convergence speed and instability [39].

Note that the main difference between [39] and our method is that we utilizing the local and nonlocal redundancies to constraint sparse coding coefficient, while method in [39] only using the local sparsity constraint. More specifically, we not only exploited the image nonlocal self-similarity to better estimate the sparse coding coefficients, but also centralized the sparse coding coefficients of the observed image to those estimates. However, [39] only utilized the image nonlocal self-similarity to estimate the local sparse coefficients, which may not an inaccurate CS reconstruction results.

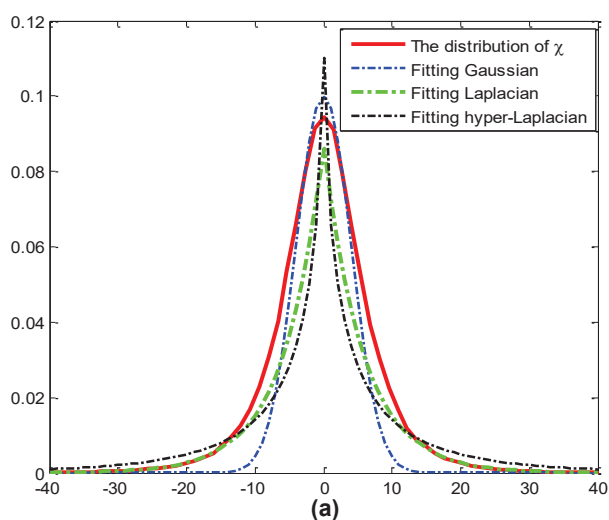

(a)

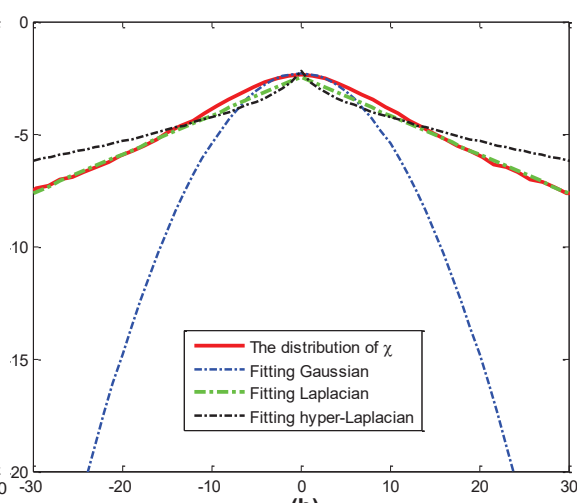

(b)

Fig. 1: The distribution of $\chi$ for image Leaves with $0.2 N$ measurements and the fitting Gaussian, Laplacian distribution, hyper-Laplacian distribution in (a) linear and (b) log domain, respectively.

In addition, based on the above analysis and due to the unknown structure of $\boldsymbol{R}(\boldsymbol{\alpha})$ in Eq.(18), we assume that the error vector $\boldsymbol{e}=\boldsymbol{x}-\boldsymbol{r}$ follows an independent zero-mean distribution with variance $\sigma^{2}$ and achieves a conclusion of Theorem 1. Later, we deduce that Eq.(18) can convert into Eq.(21) which can efficiently solve $n$ sub-problems for all the image patches $\boldsymbol{x}_{i}$. In other words, Eq.(21) can also be viewed as the sparse coding problem.

Because we know the detailed structure of $\boldsymbol{R}(\boldsymbol{\alpha})$ in Eq.(27), namely, $\boldsymbol{R}(\boldsymbol{\alpha})=$ $\|\boldsymbol{\alpha}-\boldsymbol{u}\|_{1}$, a series of experiments can be made. Specifically, because we regard $\boldsymbol{r}$ as the approximation of the original image $\boldsymbol{x}$, we achieve some experiments to survey the statistics of $\boldsymbol{e}=\boldsymbol{x}-\boldsymbol{r}$. In these experiments, a gray image Leaves is used as an example in the context of compressed sensing image reconstruction, where the original image is undersampled by a random Gaussian matrix with $0.2 N$ measurements. At each iteration $t, \boldsymbol{r}^{(t)}$ can be obtained by 


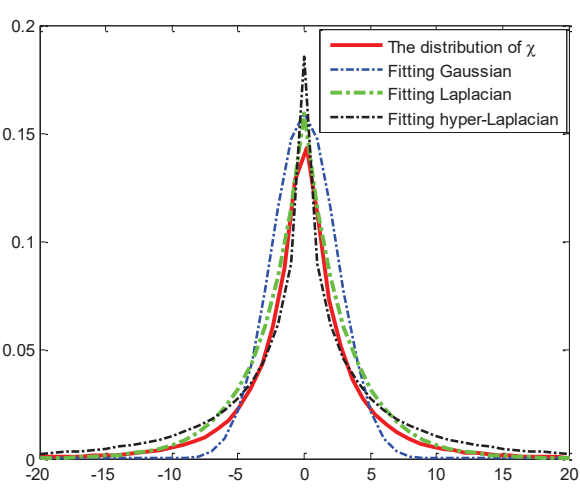

(a)

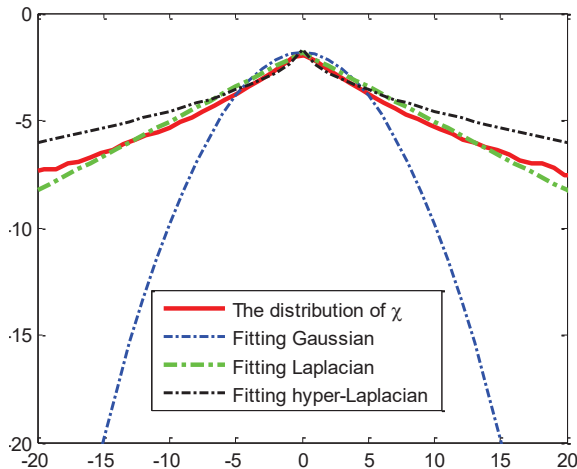

(b)

Fig. 2: The distribution of $\chi$ for image Monarch with $0.3 N$ measurements and the fitting Gaussian, Laplacian distribution, hyper-Laplacian distribution in (a) linear and (b) $\log$ domain, respectively.

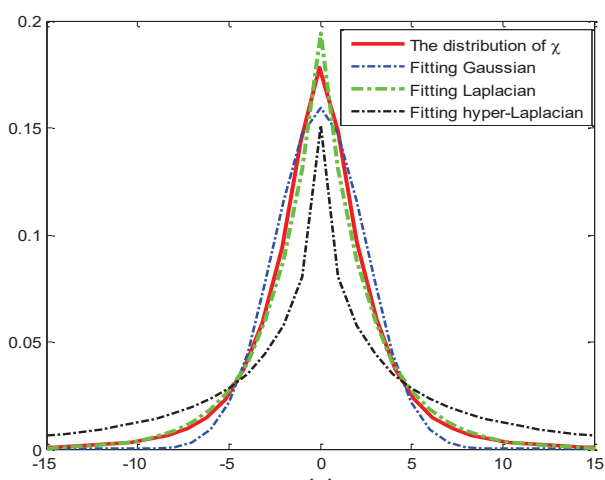

(a)

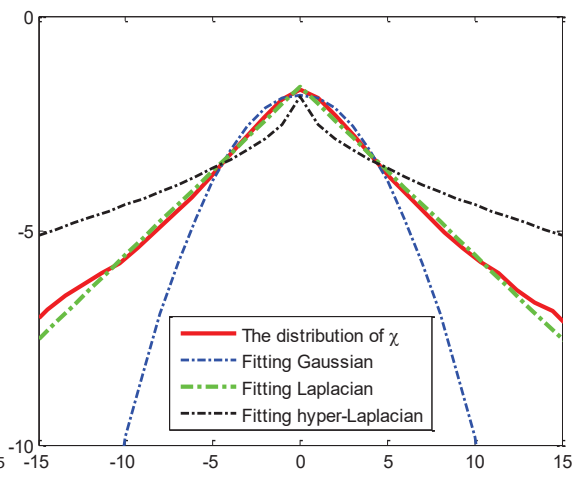

(b)

Fig. 3: The distribution of $\chi$ for image boats with $0.4 N$ measurements and the fitting Gaussian, Laplacian distribution, hyper-Laplacian distribution in (a) linear and (b) $\log$ domain, respectively.

$\boldsymbol{r}^{(t)}=\boldsymbol{z}^{(t)}-\boldsymbol{b}^{(t-1)}$. Since it is difficult to achieve the exact minimization of Eq.(27), we approximate $\boldsymbol{x}^{(t)}$ by $\boldsymbol{r}^{(t)}$ without the loss of generality. Thus, it is possible to achieve the histogram of residual $\boldsymbol{e}^{(t)}=\boldsymbol{x}^{(t)}-\boldsymbol{r}^{(t)}$ at each iteration $t$. Fig. 4 shows the distributions of residual $\boldsymbol{e}^{(t)}$ when $t$ is equal to 3 and 9 , respectively.

From Fig.4, it is clear that the distribution of $\boldsymbol{e}^{(t)}$ at each iteration is very similar to the generalized Gaussian distribution (GGD) [38] with zero-mean and variance $\sigma^{2}$. Therefore, we assume that the error vector $\boldsymbol{e}=\boldsymbol{x}-\boldsymbol{r}$ follows an independent zero-mean distribution with variance $\sigma^{2}$ available in Eq.(18). 


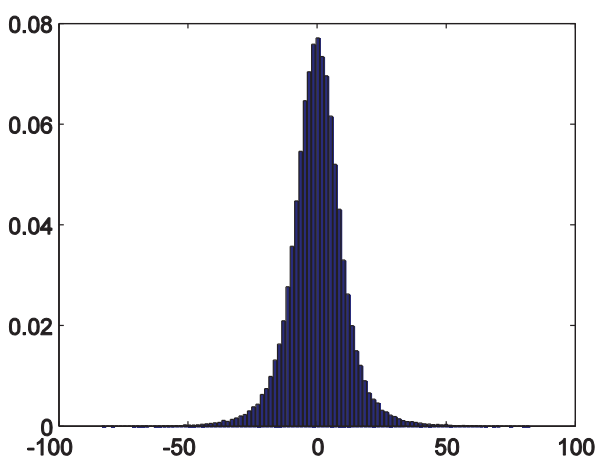

(a)

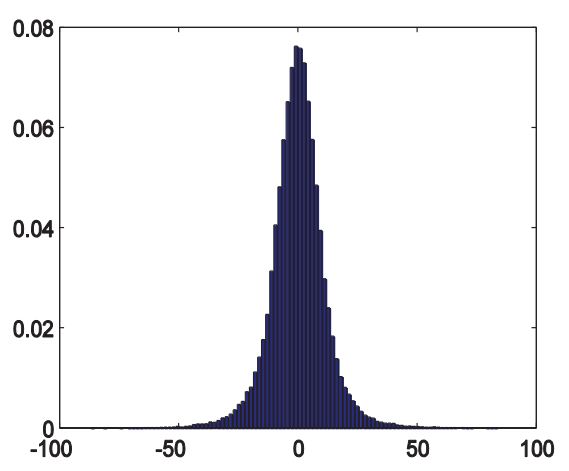

(b)

Fig. 4: The distribution of $e^{(t)}$ for image Leaves in the context of CS image reconstruction at different iteration. (a) $(t)=3 ;(\mathrm{b})(t)=9$;

\subsection{Self-Adaptive PCA dictionary learning}

Recently, there have been many works about learning dictionaries [14, 4043] from natural image patches. It is well-known that the KSVD [20,21] can represent various image local structures by learning a universal dictionary from natural image dataset. However, it has been shown that sparse coding over a highly redundant dictionary is potentially unstable and tends to generate visual artifacts [24,25]. In our method, we adopt the strategy in [14], that is, the $K$-means clustering combined with the local PCA are used to learn a set of PCA sub-dictionaries.

In order to obtain the self-adaptive PCA dictionary, the training image patches are usually derived from the natural image dataset or the original image. However, in many practical situations the training example images are simply not available, and, even in the CS image reconstruction, the original image is also not available; we can only use the CS measurements from Eq.(1). Although the original image $\boldsymbol{x}$ is unknown, we can still extract all the image patches from $r$ to achieve a self-adaptive PCA dictionary, because $r$ can be regarded as a good approximation of the original image $\boldsymbol{x}$ at each iteration in Eq.(27). We divide $\boldsymbol{r}$ into patches and cluster the patches into multiple clusters. In order to remove the redundancy from each cluster, we use the PCA to handle it to learn a local sub-dictionary. Meanwhile, the centroid of each cluster is calculated. For each given image patch, the Euclidian distance between the patch and the centroid of each sub-dictionary is calculated to adaptively choose the proper sub-dictionary. More details about the PCA dictionary can be seen in [14]. 
Table 2: Complete Distribution of proposed ASNR model for CS image reconstruction

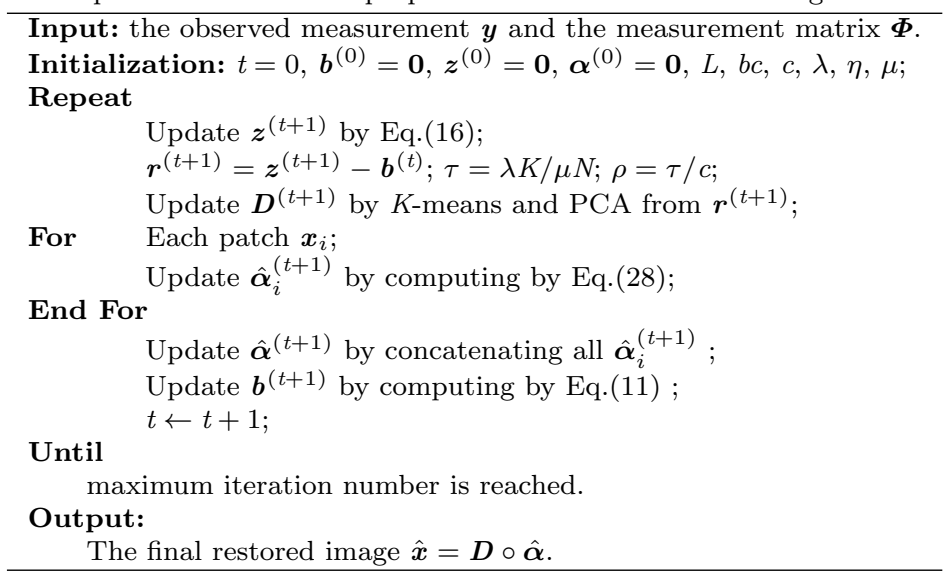

\subsection{Summary of the proposed method}

So far, the above two sub-problems have been solved. In fact, we achieve an efficient solution by solving each sub-problem separately, which can guarantee the whole method to be more efficient and effective. Based on the above derivations, the complete description of the proposed method for the CS image reconstruction via the ASNR model is provided in Table 2.

\section{Experimental Results}

4.1 Experimental framework and parameters setting

In this section, we will report the experimental results of the proposed ASNRbased CS image reconstruction method. We generated the CS measurements at the block level by utilizing the Gaussian random projection matrix to test images, i.e., the block-based CS recovery with block size of $32 \times 32$. In our experiments, both the natural images and the MRI images were used to verify the performance of the proposed ASNR method. The main parameters of the proposed method were set as follows: the size of each patch, i.e., $\sqrt{b c} \times \sqrt{b c}$ was $6 \times 6, \mu$ was set to be $0.0025, \eta$ was set to be 1 , and the total $L=40$ matched patches were selected for each exemplar patch. The parameter $\lambda$ that balances the fidelity term and the regularization term should be tuned separately for each sensing rate. The test images which were used in our experiments consisted of eight natural images and two MRI images of size $256 \times 256$ as shown in Fig.5. The platform for the comparison was an Intel(R) Core(TM) i3-4150 with a $3.50 \mathrm{GHz} \mathrm{CPU}$ and $4 \mathrm{~GB}$ memory, and Windows 7 operating system. 


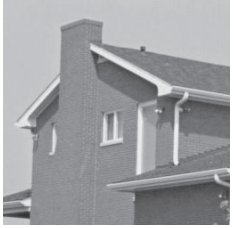

(a)

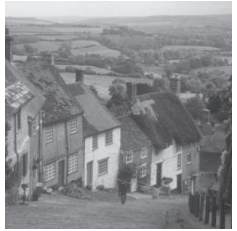

(f)

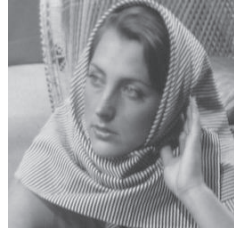

(b)

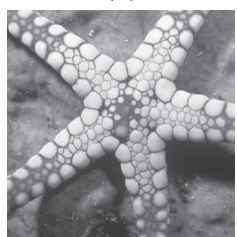

(g)

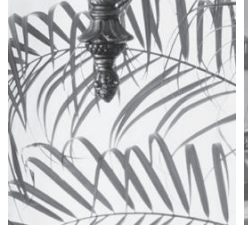

(c)

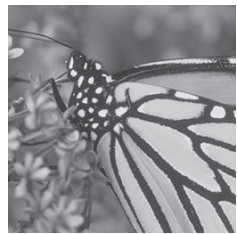

(h)

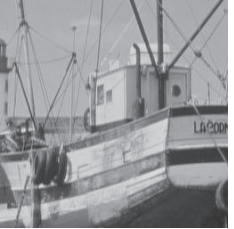

(d)

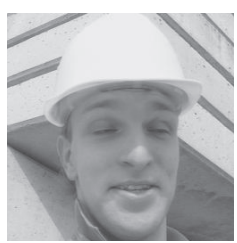

(e)

Fig. 5: Test images used for compressed sensing reconstruction experiments. (a) House. (b) Barbara. (c) Leaves. (d) boats. (e) foreman. (f) Goldhill. (g) starfish. (h) Monarch. (i) Brain MRI image. (j) Bone MRI image.

\subsection{Numerical performance comparisons}

To verify the performance of the proposed ASNR method, we compared it with several competitive CS recovery methods including the BCS method [44], the NESTA method [45], the COS method [46], the BM3D based CS recovery method [47] (denoted as the BM3D-CS), the ASD-CS method [39], the NCSR-CS method [19], the adaptively learned sparsifying based CS recovery via minimization [48] (denoted as the ALSB), and the MRK-CS method [49]. The source codes of all bench methods [19,39,46-49] were obtained from the authors' websites. To have a fair comparison among the competing methods, we used the built-in parameter settings in each implementation which performed optimally.

In the first experiment, the numerical performance comparison of the proposed ASNR method was as follows. The PSNR comparison results of recovered images by competing CS recovery methods are shown in Table 3 . The ASNR method consistently outperformed other methods on most of the test images over different numbers of CS measurements, which further increases the performance advantage of the proposed ASNR method (e.g., the ASNR can outperform eight comparative methods by up to $6.75 \mathrm{~dB}, 5.16 \mathrm{~dB}, 3.90 \mathrm{~dB}$, $2.52 \mathrm{~dB}, 0.66 \mathrm{~dB}, 0.58 \mathrm{~dB}, 1.77 \mathrm{~dB}$ and $1.22 \mathrm{~dB}$, respectively). On average, the proposed ASNR method outperforms all previous benchmark methods. The PSNR gains of the ASNR over the BCS, NESTA, COS, BM3D-CS, ASD-CS, NCSR-CS, ALSB, and MRK-CS can be as much as $9.70 \mathrm{~dB}, 9.83 \mathrm{~dB}, 5.74 \mathrm{~dB}$, $4.15 \mathrm{~dB}, 1.06 \mathrm{~dB}, 0.71 \mathrm{~dB}, 1.32 \mathrm{~dB}$ and $3.34 \mathrm{~dB}$, respectively on Barbara image the most favorable situation for an adaptive sparse nonlocal regularization and the split Bregman iteration algorithm is exploited. In many cases, it has to be pointed out that the gain of the proposed ASNR method with low sampling ratios is larger than with high sampling ratios. The reason for this is that the 
self-adaptive PCA dictionary learning method can capture the local image feature better and greatly reduce visual artifacts in the case of low sampling ratios than some predefined transform and some over-complete dictionary learning methods.

\subsection{Visual quality comparisons}

To facilitate the evaluation of subjective qualities, some of the reconstructed images are shown in Figs.6-11. From them, we can see that the BCS and NESTA methods cannot reconstruct sharp edges and fine image details. The COS, BM3D-CS, ASD-CS, NCSR-CS, ALSB and MRK-CS methods can generate better visual quality than the BCS and NESTA methods. However, the COS, BM3D-CS and NCSR-CS still suffer from some undesirable over-smooth phenomena, such as the loss of some fine image details and visual artifacts that can still be clearly observed from the ASD-CS, ALSB and MRK-CS. By contrast, the proposed ASNR approach not only removes most of the visual artifacts and ring effects, but also effectively preserves large-scale sharp edges and smallscale fine image details in comparison with the other competing methods. The high performance of the proposed ASNR approach is attributed to the following facts: First, to exploit the self-adaptive PCA sub-dictionary can effectively capture the local image feature to greatly reduce visual artifacts. Second, an adaptive sparse nonlocal regularization can be introduced to further enhance the CS image reconstruction performance. Third, the split Bregman iteration algorithm is developed to improve the computational efficiency of the proposed ASNR method.

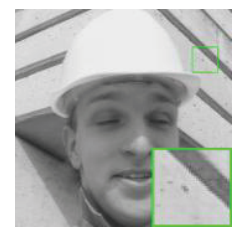

(a)

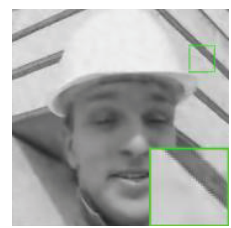

(f)

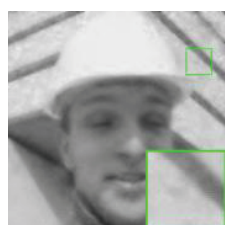

(b)

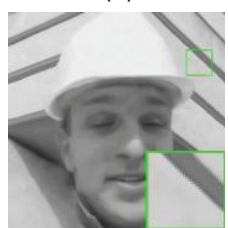

(g)

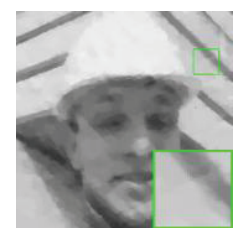

(c)

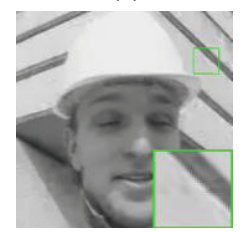

(h)

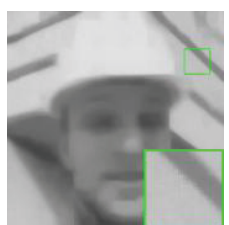

(d)

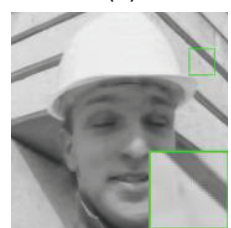

(i)

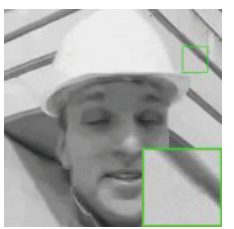

(e)

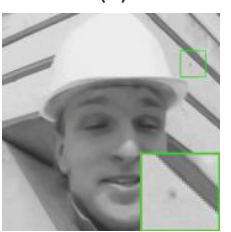

(j)

Fig. 6: CS reconstruction foreman image with $0.1 N$ measurements. (a) original image; (b) BCS reconstruction [44] (29.76dB); (c) NESTA reconstruction [45] (29.94dB); (d) COS reconstruction [46] (24.66dB); (e) BM3D-CS reconstruction [47] (32.68dB); (f) ASD-CS reconstruction [39] (35.03dB); (g) NCSR-CS reconstruction [19] (34.18dB); (h) ALSB reconstruction [48] (34.34dB); (i) MRK-CS reconstruction [49] (35.29dB); (j) Proposed ASNR reconstruction (36.11dB). 


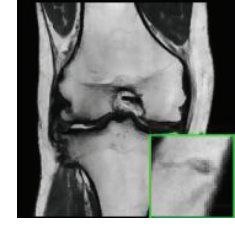

(a)

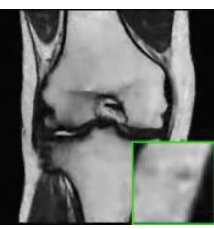

(f)

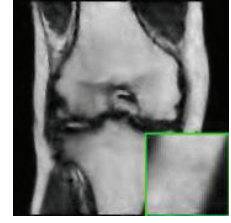

(b)

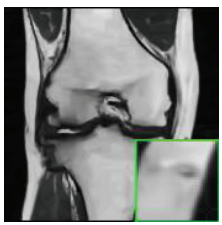

(g)

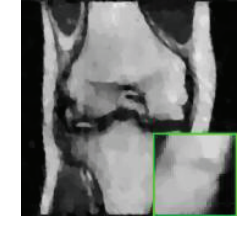

(c)

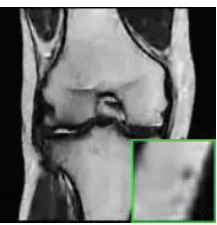

(h)

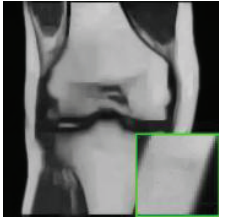

(d)

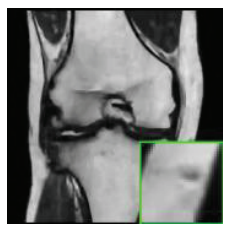

(i)

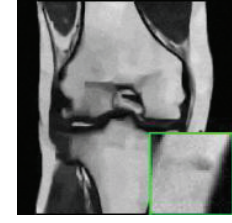

(e)

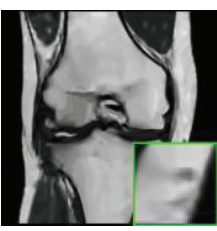

(j)

Fig. 7: CS reconstruction Bone image with $0.1 N$ measurements. (a) original image; (b) BCS reconstruction [44] (24.93dB); (c) NESTA reconstruction [45] (24.82dB); (d) COS reconstruction [46] (24.49dB); (e) BM3D-CS reconstruction [47] (27.39dB); (f) ASD-CS reconstruction [39] (29.23dB); (g) NCSR-CS reconstruction [19] (29.14dB); (h) ALSB reconstruction [48] (27.93dB); (i) MRK-CS reconstruction [49] (29.09dB); (j) Proposed ASNR reconstruction $(29.38 \mathrm{~dB})$.

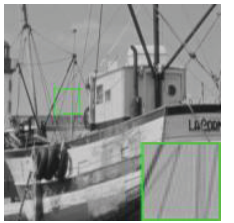

(a)

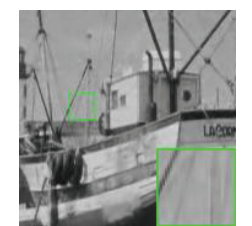

(f)

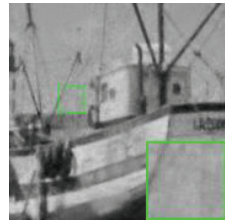

(b)

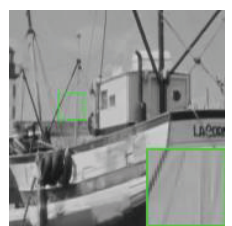

(g)

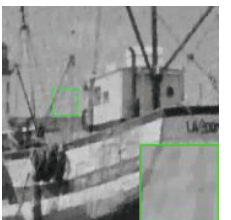

(c)

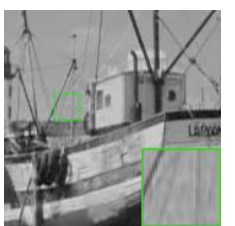

(h)

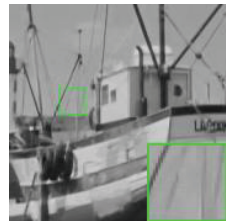

(d)

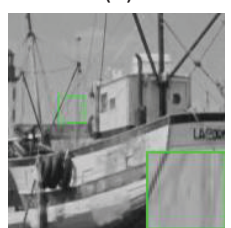

(i)

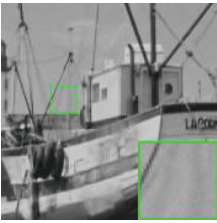

(e)

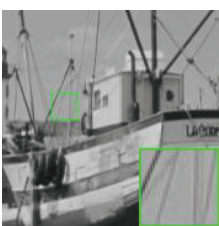

(j)

Fig. 8: CS reconstruction boats image with $0.2 N$ measurements. (a) original image; (b) BCS reconstruction [44] (27.05dB); (c) NESTA reconstruction [45] (28.30dB); (d) COS reconstruction [46] (31.43dB); (e) BM3D-CS reconstruction [47] (30.99dB); (f) ASD-CS reconstruction [39] (33.15dB); (g) NCSR-CS reconstruction [19] (33.56dB); (h) ALSB reconstruction [48] (31.92dB); (i) MRK-CS reconstruction [49] (32.37dB); (j) Proposed ASNR reconstruction (33.63dB). 


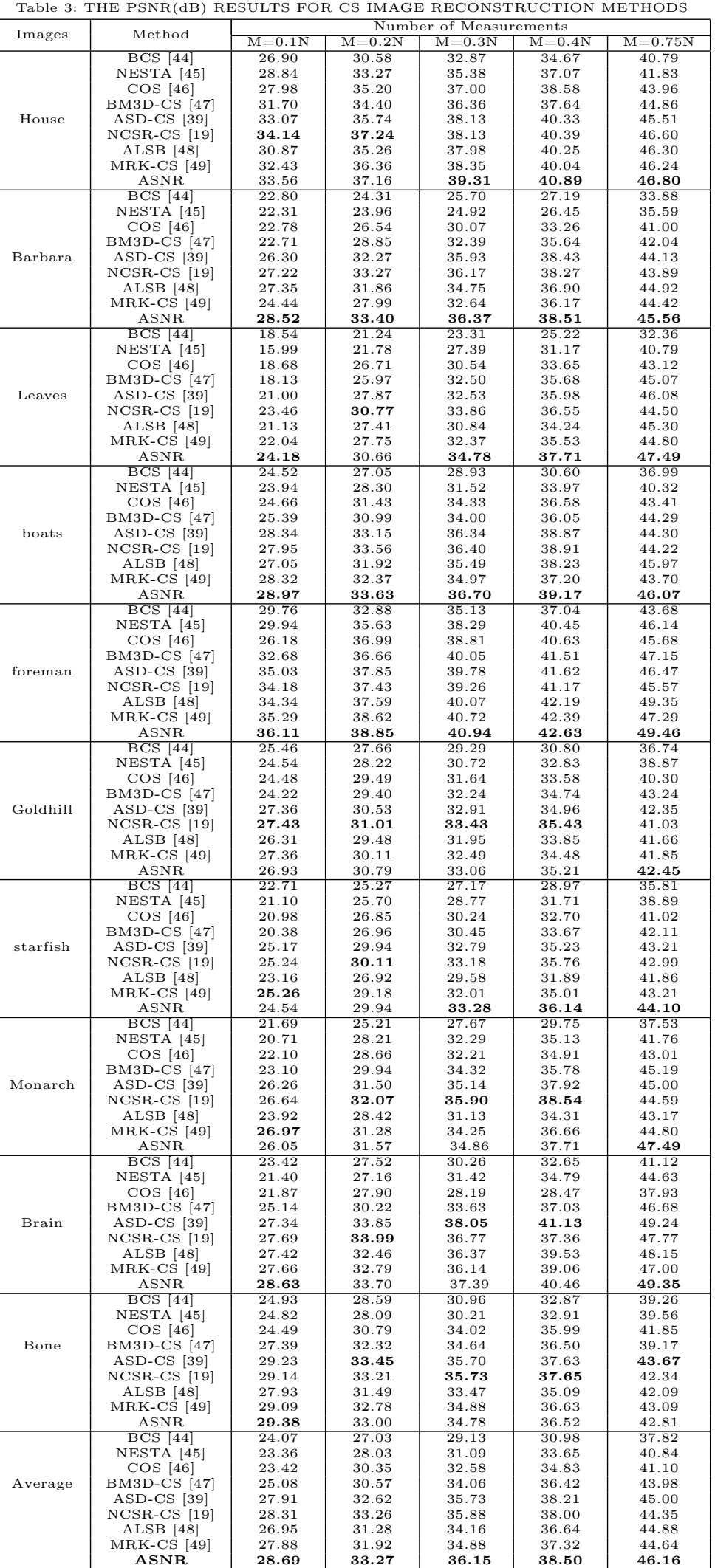




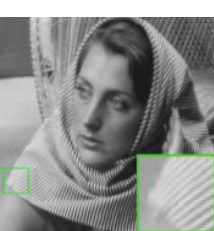

(a)

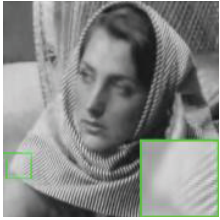

(f)

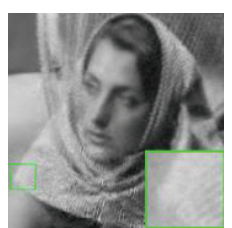

(b)

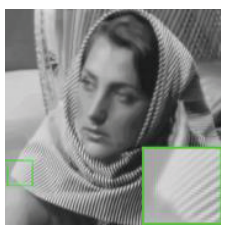

(g)

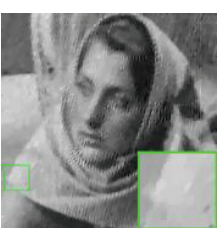

(c)

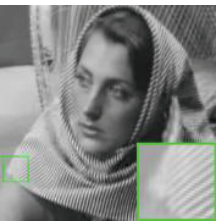

(h)

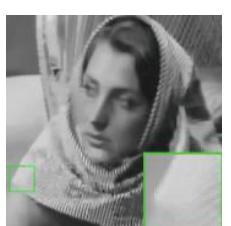

(d)

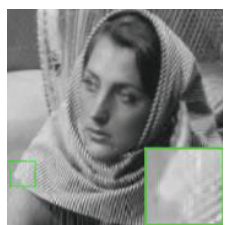

(i)

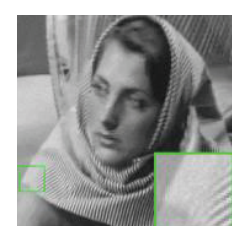

(e)

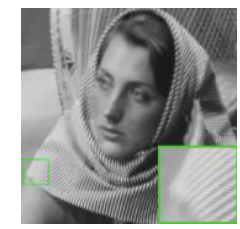

(j)

Fig. 9: CS reconstruction Barbara image with $0.2 N$ measurements. (a) original image; (b) BCS reconstruction [44] (24.31dB); (c) NESTA reconstruction [45] (23.96dB); (d) COS reconstruction [46] (26.54dB); (e) BM3D-CS reconstruction [47] (28.85dB); (f) ASD-CS reconstruction [39] (32.27dB); (g) NCSR-CS reconstruction [19] (33.27dB); (h) ALSB reconstruction [48] (31.86dB); (i) MRK-CS reconstruction [49] (27.99dB); (j) Proposed ASNR reconstruction $(33.40 \mathrm{~dB})$.

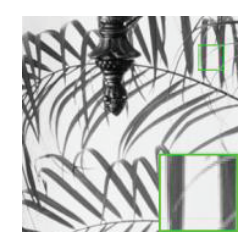

(a)

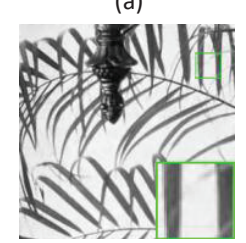

(f)

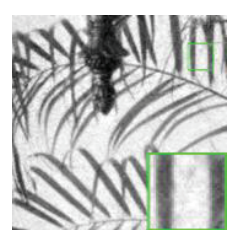

(b)

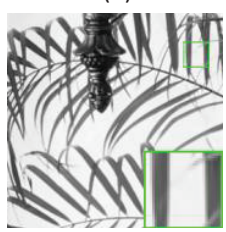

(g)

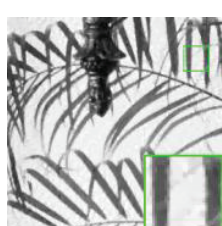

(c)

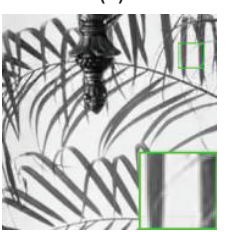

(h)

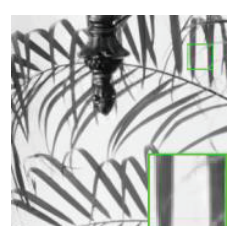

(d)

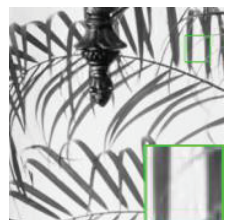

(i)

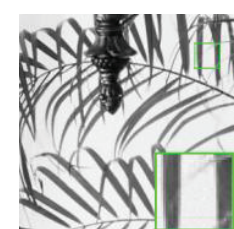

(e)

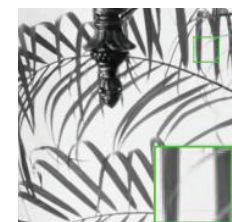

Fig. 10: CS reconstruction Leaves image with $0.3 N$ measurements. (a) original image; (b) BCS reconstruction [44] (23.31dB); (c) NESTA reconstruction [45] (27.39dB); (d) COS reconstruction [46] (30.54dB); (e) BM3D-CS reconstruction [47] (32.50dB); (f) ASD-CS reconstruction [39] (32.53dB); (g) NCSR-CS reconstruction [19] (33.86dB); (h) ALSB reconstruction [48] (30.84dB); (i) MRK-CS reconstruction [49] (32.37dB); (j) Proposed ASNR reconstruction $(\mathbf{3 4 . 7 8} \mathrm{dB})$.

\subsection{Effect of the dictionary selection}

For this sub-section, another experiment was conducted to further evaluate the influence of different dictionary on the proposed ASNR reconstruction method. All the parameters were the same as specified in Section 4.1, except for the usage of the proposed dictionaries, including the standard DCT dictionary (SDCT), over-complete DCT dictionary (ODCT), and the global- 


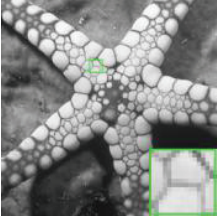

(a)

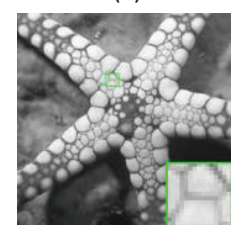

(f)

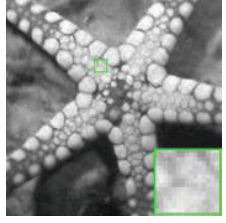

(b)

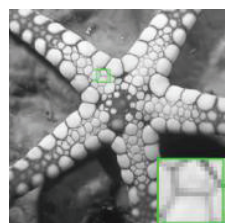

(g)

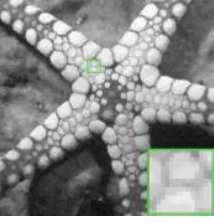

(c)

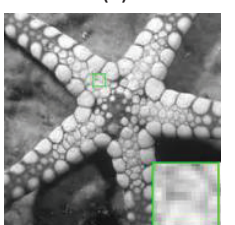

(h)

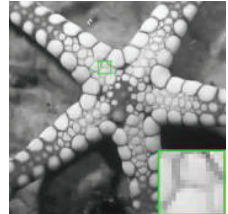

(d)

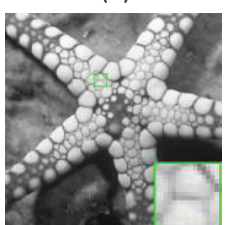

(i)

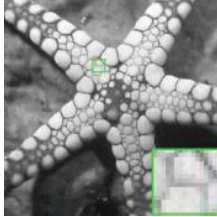

(e)

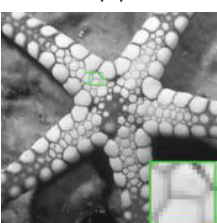

(j)

Fig. 11: CS reconstruction starfish image with $0.3 N$ measurements. (a) original image; (b) BCS reconstruction [44] (27.17dB); (c) NESTA reconstruction [45] (28.77dB); (d) COS reconstruction [46] (30.24dB); (e) BM3D-CS reconstruction [47] (30.45dB); (f) ASD-CS reconstruction [39] (32.79dB); (g) NCSR-CS reconstruction [19] (33.18dB); (h) ALSB reconstruction [48] (29.58dB); (i) MRK-CS reconstruction [49] (32.01dB); (j) Proposed ASNR reconstruction $(\mathbf{3 3 . 2 8} \mathrm{dB})$.

ly trained dictionary (GDIC), as shown in Fig.12. For different dictionaries, the CS reconstruction results for the image Starfish with $0.3 \mathrm{~N}$ measurements are shown in Fig.13. It is obvious that the reconstruction error images of the proposed self-adaptive PCA dictionary have less texture features than those of other dictionary learning methods, as shown in Fig.13 (g)-(j) and Fig.13 (l)-(o), respectively. In other words, the proposed self-adaptive PCA learning dictionary method generates better results than other competing dictionary learning methods. The reason is that over-complete redundant dictionary is potentially unstable and tends to generate visual artifacts, while the proposed PCA sub-dictionary can capture the local image feature better and greatly reduce artifacts. In addition, we compare the proposed PCA sub-dictionary, which is learned from natural image dataset and the reconstructed image itself. We use the training dataset 2 as natural image dataset in [14]. From Fig.14 and Fig.15, we can see that the dictionary learned from natural image dataset loses of the fine details, while the dictionary learned from the reconstructed image itself can obtain fine details and produce more effective results than the dictionary learned from natural image dataset. The reason is that the natural image dataset can not always give fine description for various matching textures. However, the similar texture can be found in the reconstructed image without any doubt.

\subsection{Effect of the number of best matched patches}

In this sub-section, we will discuss how to select the best-matching patch numbers $L$ for enhancing the performance of the proposed ASNR method. 


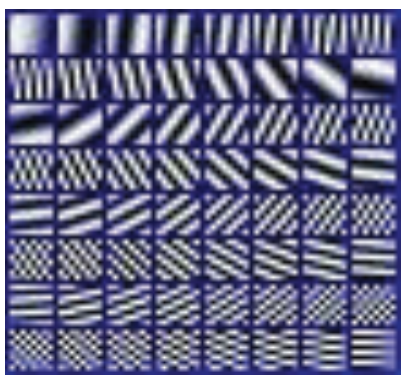

(a)

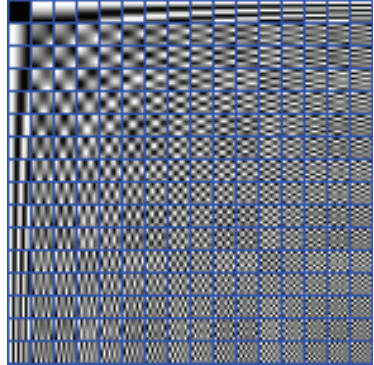

(b)

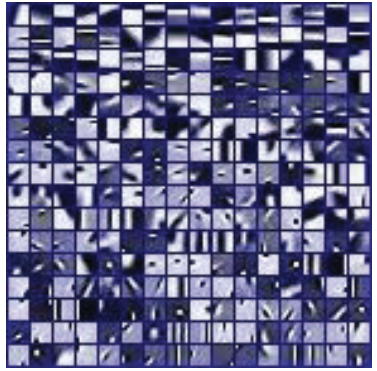

(c)

Fig. 12: Three different types of dictionaries. (a) SDCT. (b) ODCT. (c) GDIC.

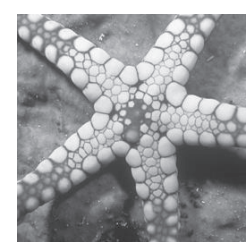

(a)

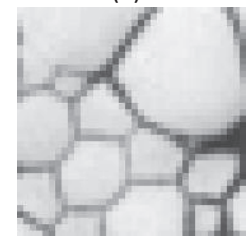

(f)

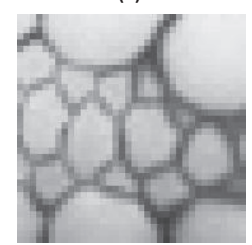

(k)

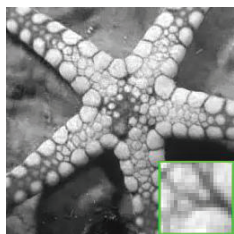

(b)

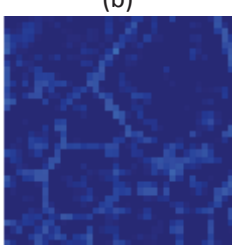

(g)

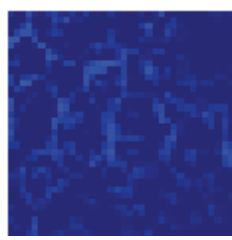

(I)

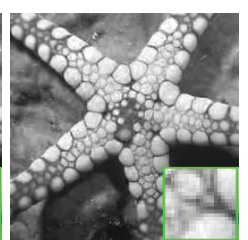

(c)

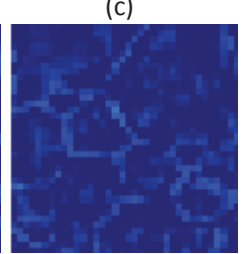

(h)

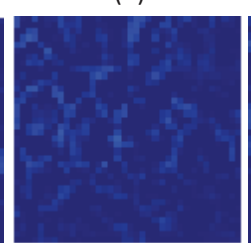

(m)

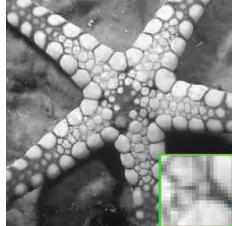

(d)

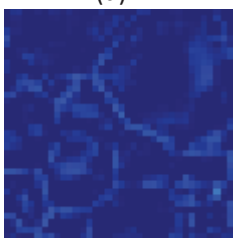

(i)

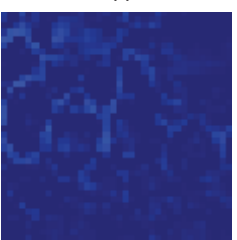

(n)

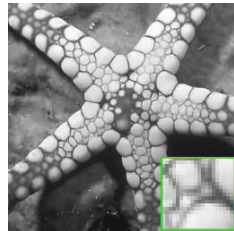

(e)

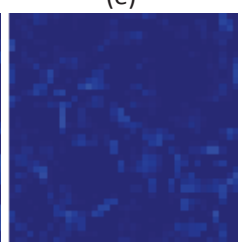

(j)

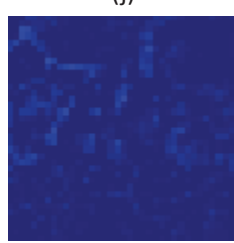

(o)

Fig. 13: CS reconstruction Starfish image with $0.3 N$ measurements by different dictionaries. (a) original image; (b) SDCT reconstruction $(28.22 \mathrm{~dB}$ ); (c) DCT reconstruction $(29.21 \mathrm{~dB})$; (d) GDIC reconstruction $(30.19 \mathrm{~dB})$; (e) Proposed self-adaptive PCA dictionary reconstruction $(\mathbf{3 3 . 2 8} \mathrm{dB})$; (f) and $(\mathrm{k})$ different local magnification patch of the original image, respectively. (g-j) and (l-o) are the reconstruction error images by (f) and $(\mathrm{k})$ with different dictionaries, respectively.

Specifically, to investigate the sensitivity of $L$, we conducted two experiments on three test images with respect to different $L$, ranging from 20 to 140 , in the case of $0.1 \mathrm{~N}$ measurement and $0.3 \mathrm{~N}$ measurement, respectively. The performance comparison with different $L$ is shown in Fig.16. It is clear to see that all the curves are almost flat in Fig.16, in other words, the performance of the proposed ASNR method is not too sensitive to $L$. The best performance 


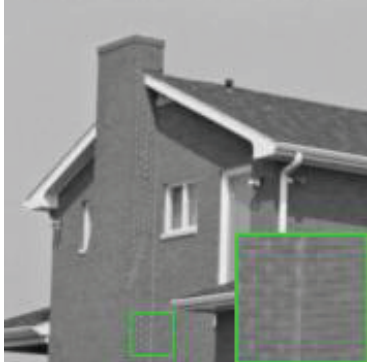

(a)

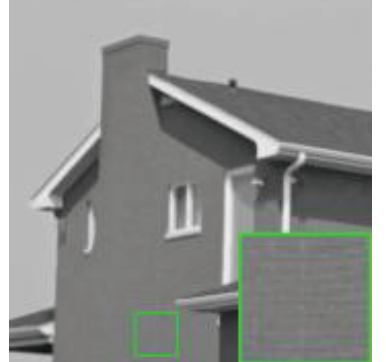

(b)

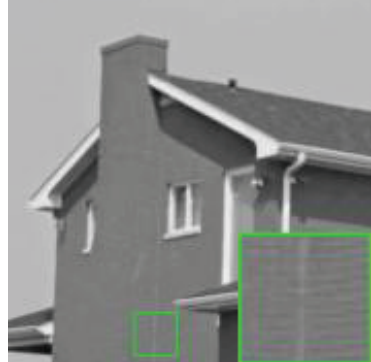

(c)

Fig. 14: CS reconstruction House image with $0.2 N$ measurements by different dictionaries.

(a) original image; (b) Dictionary learning from natural image dataset (35.56dB); (c) Dictionary learning from the reconstructed image itself (37.16dB).

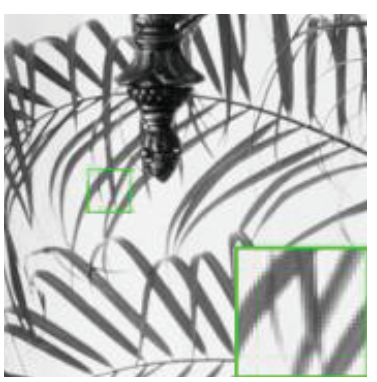

(a)

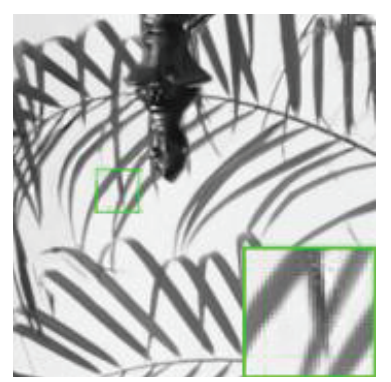

(b)

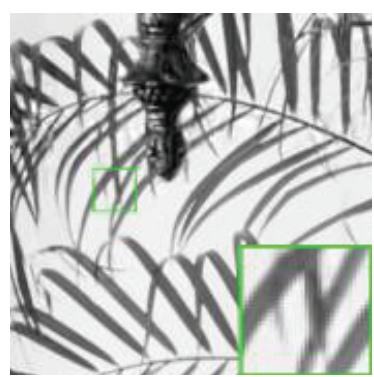

(c)

Fig. 15: CS reconstruction Leaves image with $0.2 N$ measurements by different dictionaries. (a) original image; (b) Dictionary learning from natural image dataset $(28.14 \mathrm{~dB})$; (c) Dictionary learning from the reconstructed image itself $(\mathbf{2 8 . 8 9} \mathrm{dB})$.

of each case was usually achieved with $L$ in the range $[20,60]$. Thus, $L$ was empirically set to be 40 .

\subsection{Comparison of the noise level for different methods}

To test the noise sensitivity of the proposed ASNR method, we first conducted experiments with zero-mean Gaussian white noise added to the compressed sensing measurements. The small noise level was 1e-3, 2e-3, 3e-3, 4e-3 and 5e-3. Fig. 17 shows the PSNR versus noise level in test images depicting boats and Brain under five under-sampling ratios $(0.1,0.2,0.3,0.4$, and 0.75$)$. It is obvious that the PSNR of the reconstruction image remains stable for all specified noise levels, in other words, the proposed ASNR method can adequately remove the aliasing and noise in the reconstructed image since sparse coding based on an self-adaptive PCA dictionary and an adaptive sparse nonlocal regularization has the intrinsic denoising property. In addition, we conduct 


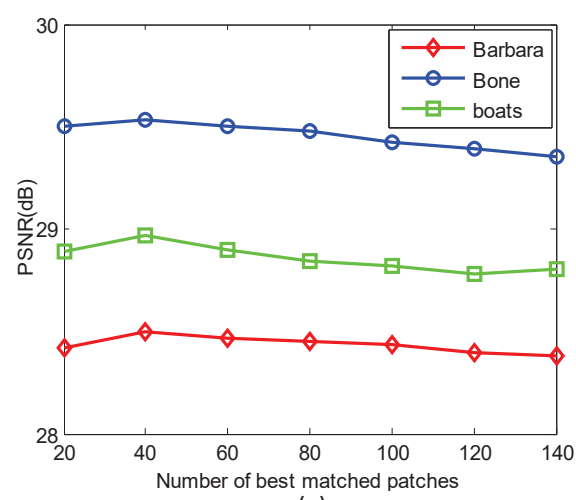

(a)

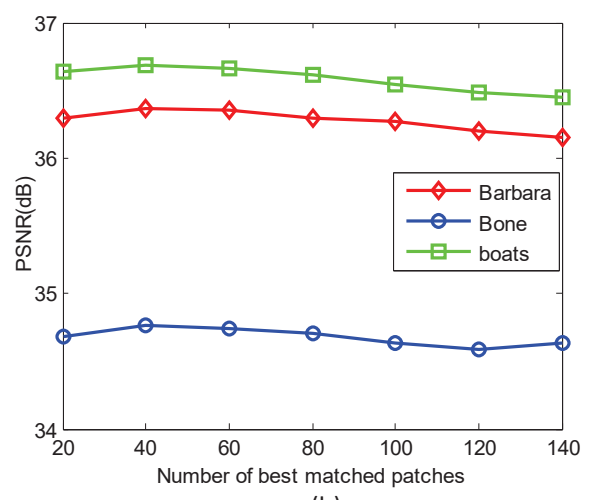

(b)

Fig. 16: Performance comparison with different matched patch numbers $L$ for three test images. (a) the PSNR (dB) results achieved by different $L$ in the case of $0.1 \mathrm{~N}$

measurement. (b) the PSNR (dB) results achieved by different $L$ in the case of $0.3 N$ measurement.

similar experiments with noisy CS measurements to demonstrate the robustness of the proposed ASNR under large noise situation. A significant amount of additive white Gaussian noise was added to the CS measurements. The standard derivations of additive noise vary to generate signal-to-noise-ratio (SNR) between $10 \mathrm{~dB}, 15 \mathrm{~dB}, 20 \mathrm{~dB}, 25 \mathrm{~dB}$ and $30 \mathrm{~dB}$. All the parameters were the same as specified in Section 4.1, except $\mu=19,9,3.5,2.5$ and 0.25 corresponding to $\mathrm{SNR}=10 \mathrm{~dB}, 15 \mathrm{~dB}, 20 \mathrm{~dB}, 25 \mathrm{~dB}$ and $30 \mathrm{~dB}$, respectively. In these experiments, the NESTA and MRK-CS methods have not included since they are sensitive to noise. The PSNR comparison of the reconstructed images are shown in Fig.18. One can observe that the proposed method outperforms other competing methods in all situations and subjective quality comparison results with $\mathrm{SNR}=20 \mathrm{~dB}, 15 \mathrm{~dB}, 10 \mathrm{~dB}$ for image Barbara, Bone, starfish are shown in Figs.19-21, respectively. It is obvious that the proposed ASNR method achieves the best performance to suppress noise among all the competing methods. Therefore, the proposed ASNR method is robust to noise measurement.

\subsection{Comparsion Between SBI and IST}

In this sub-section, another classical optimization method, the iterative shrinkage/thresholding (IST) [50] is exploited to solve our proposed ASNR model. We compared SBI with IST by setting ratio $=0.2$ and ratio $=0.3$ on test image foreman and Barabar. Fig.22 shows progression curves of the PSNR (dB) results. It can be seen that SBI method only requires 15 iterations (about $59 \mathrm{~s}$ per iteration) to achieve convergence, while IST needs 30 iterations (about 58s per iteration). Furthermore, the SBI method can achieve better PSNR results than IST method. Therefore, compared to IST method, the proposed SBI method can achieve better convergence speed with fewer computations. 


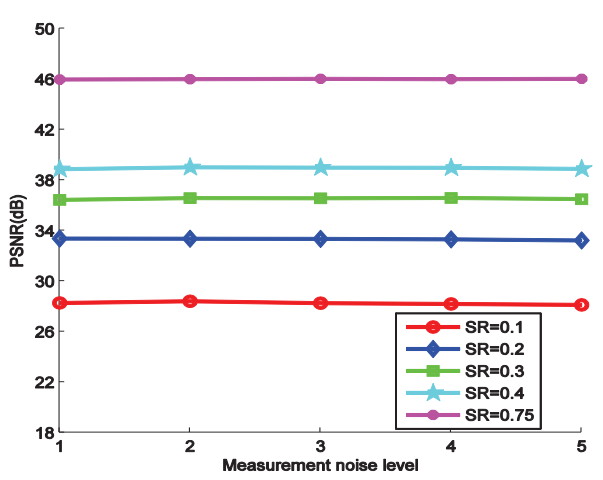

(a)

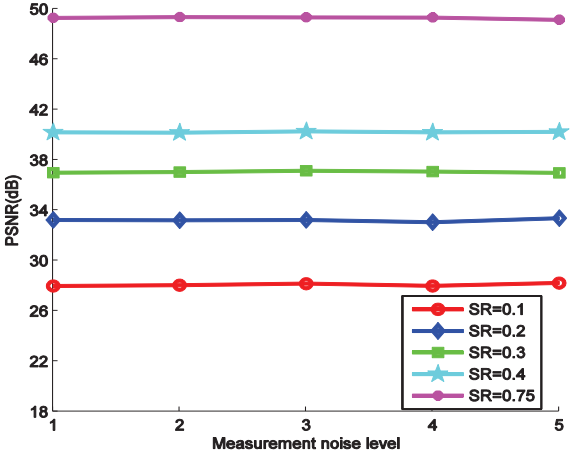

(b)

Fig. 17: Noise sensitivity analysis of the proposed ASNR method. (a) PSNR results versus measurement noise level for boats. (b) PSNR results versus measurement noise level for Brain.

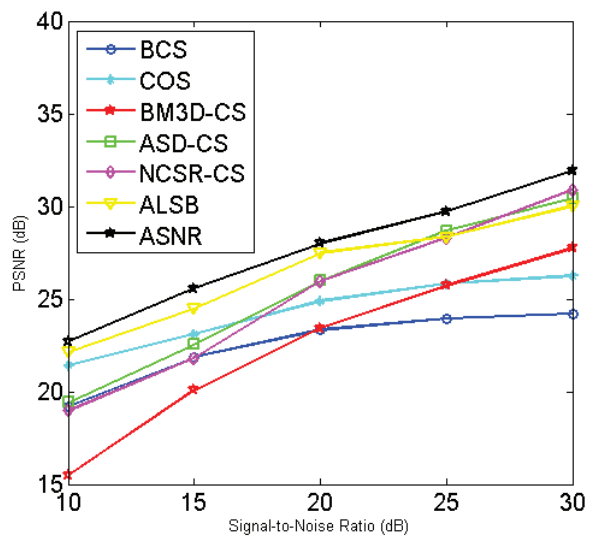

(a)

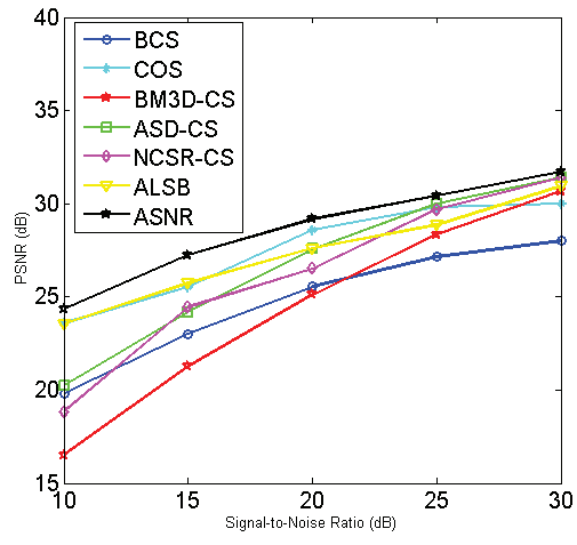

(b)

Fig. 18: PSNR of the different methods from noisy levels. (a) PSNR results versus SNR with $0.2 N$ measurements for image Barbara. (b) PSNR results versus SNR with $0.2 N$ measurements for image Bone.

\subsection{Convergence analysis}

To elaborate the convergence performance of the proposed ASNR method, Fig.23 shows the evolutions of the PSNR versus the iteration numbers for the test images depicting boats and Bone with various sampling ratios. It can be observed that with the growth of the iteration number, the PSNR curve of the reconstructed images gradually increases and ultimately tends to be flat and stable. Therefore, the good convergence performance of the proposed ASNR method is exhibited in Fig.23. 


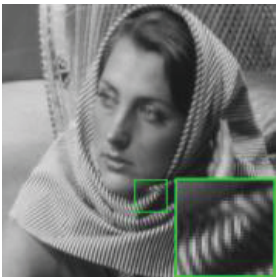

(a)

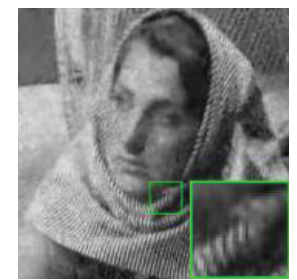

(e)

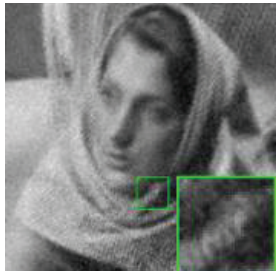

(b)

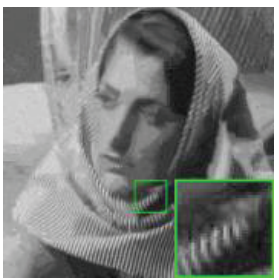

(f)

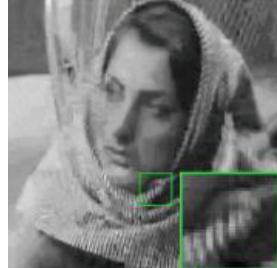

(c)

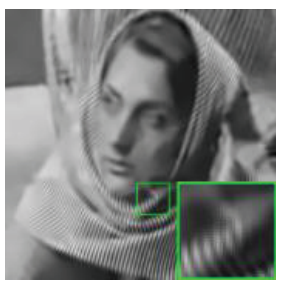

(g)

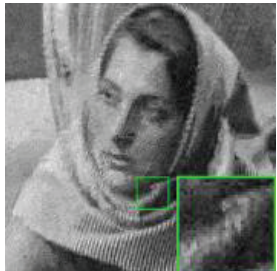

(d)

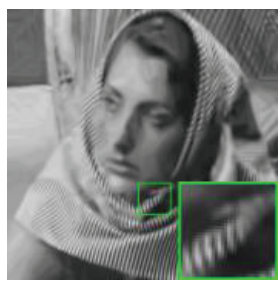

(h)

Fig. 19: Denoising performance comparison on the Barbara image with SNR=20dB. (a) Original image; (b) BCS reconstruction [44] (23.31dB); (c) COS reconstruction [46] (24.88dB); (d) BM3D-CS reconstruction [47] (23.41dB); (e) ASD-CS reconstruction [39]

(26.00dB); (f) NCSR-CS reconstruction [19] (25.95dB); (g) ALSB reconstruction [48] (27.48dB); (h) ASNR reconstruction (28.00dB).

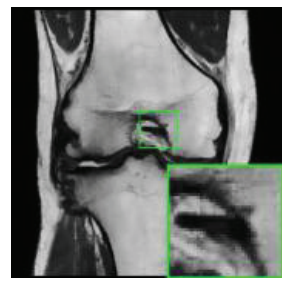

(a)

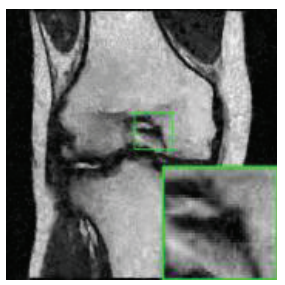

(e)

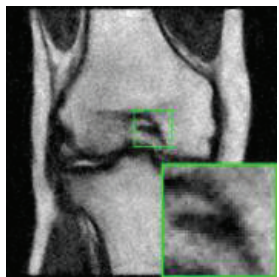

(b)

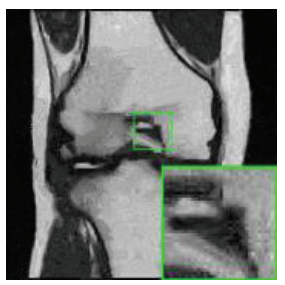

(f)

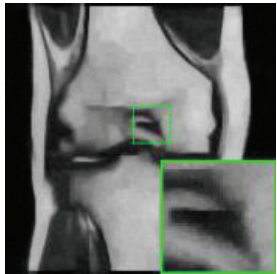

(c)

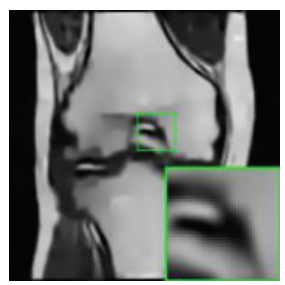

(g)

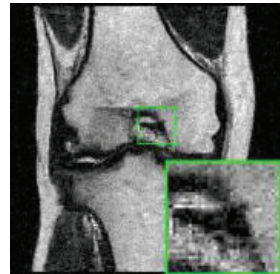

(d)

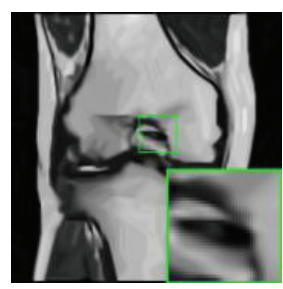

(h)

Fig. 20: Denoising performance comparison on the Bone image with $\mathrm{SNR}=15 \mathrm{~dB}$. (a) Original image; (b) BCS reconstruction [44] (23.02dB); (c) COS reconstruction [46] (25.53dB); (d) BM3D-CS reconstruction [47] (21.27dB); (e) ASD-CS reconstruction [39] (24.19dB); (f) NCSR-CS reconstruction [19] (24.45dB); (g) ALSB reconstruction [48] (25.75dB); (h) ASNR reconstruction (27.23dB).

\section{Conclusion}

In this paper, we have presented a new method toward CS image reconstruction based on adaptive sparse nonlocal regularization. The approach utilizes 


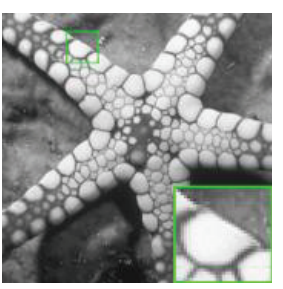

(a)

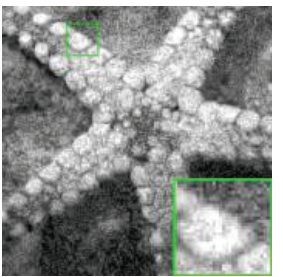

(e)

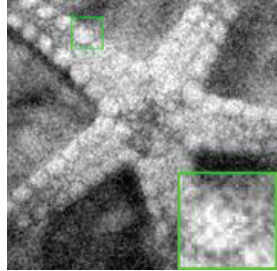

(b)

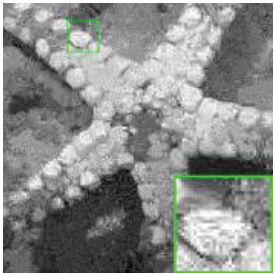

(f)

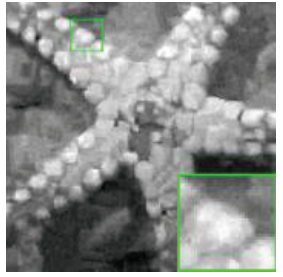

(c)

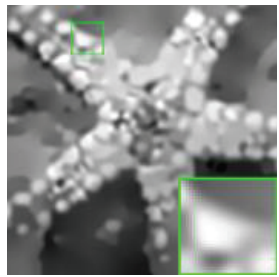

(g)

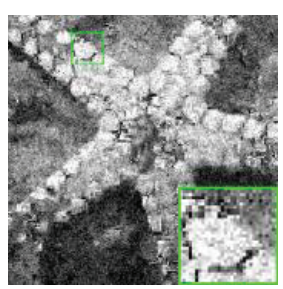

(d)

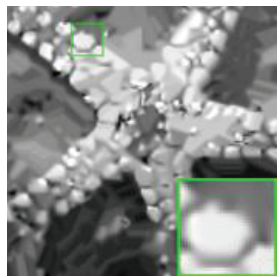

(h)

Fig. 21: Denoising performance comparison on the starfish image with $\mathrm{SNR}=10 \mathrm{~dB}$. (a) Original image; (b) BCS reconstruction [44] (18.68dB); (c) COS reconstruction [46] (21.15dB); (d) BM3D-CS reconstruction [47] (15.30dB); (e) ASD-CS reconstruction [39] (19.03dB); (f) NCSR-CS reconstruction [19] (18.88dB); (g) ALSB reconstruction [48] (20.98dB); (h) ASNR reconstruction (21.31dB).

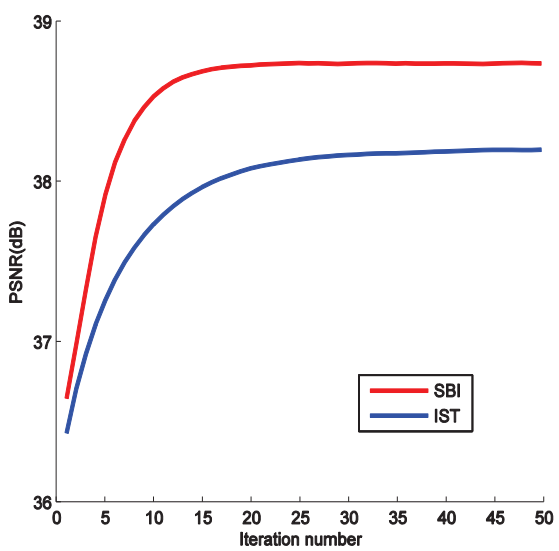

(a)

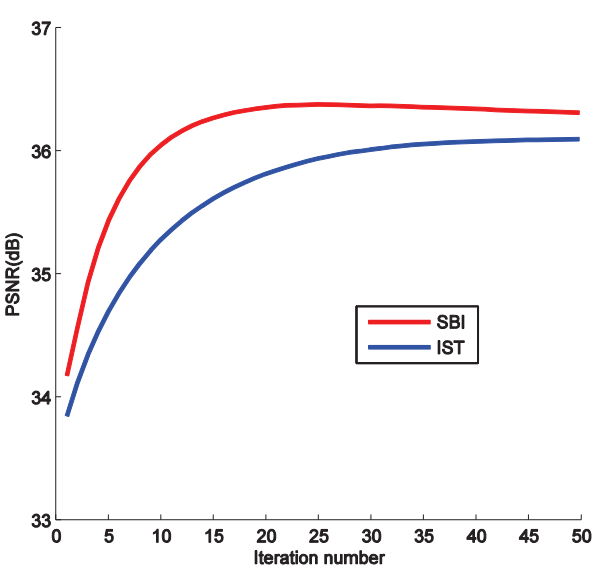

(b)

Fig. 22: Comparison between SBI and IST. (a) PSNR results achieved by SBI and IST with ratio $=0.2$ for image foreman. (b) PSNR results achieved by SBI and IST with ratio $=0.3$ for image Barbara.

the nonlocal self-similarity prior integrated into an adaptive regularization to improve the CS reconstruction performance. Meanwhile, an self-adaptive PCA sub-dictionary is exploited to effectively capture the local image feature and greatly reduce artifacts. Furthermore, we have developed a convenient implementation using the split Bregman iteration algorithm to optimize the 


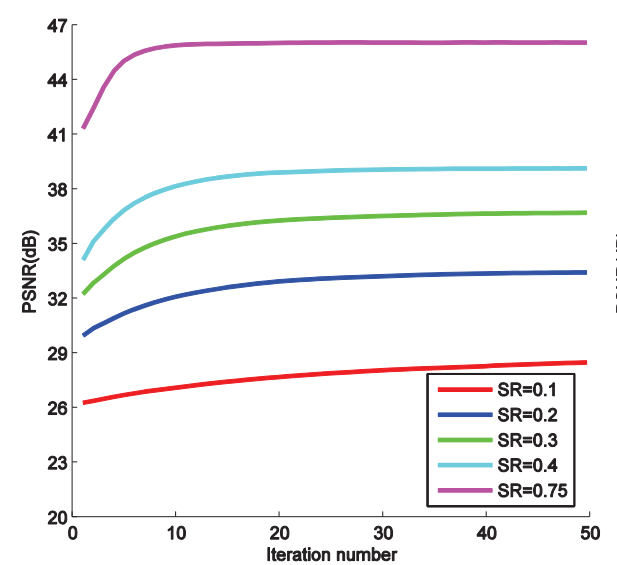

(a)

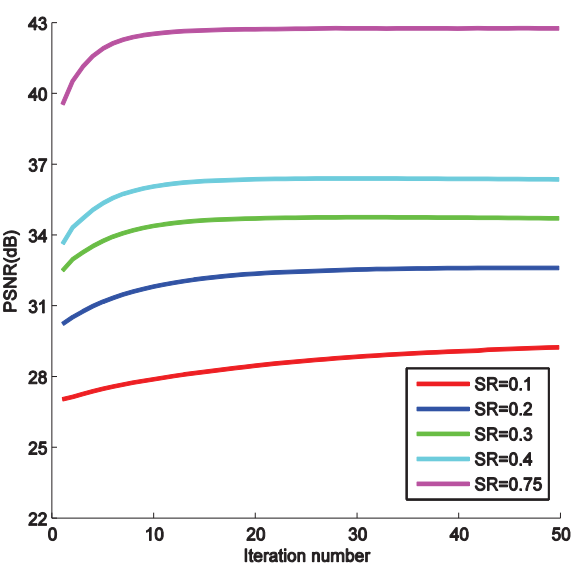

(b)

Fig. 23: Convergence analysis of the proposed ASNR method. (a) PSNR results versus iteration numbers for boats. (b) PSNR results versus iteration numbers for Bone.

proposed ASNR model, which can improve the computational efficiency and achieve good convergence property. The results clearly demonstrate that the proposed ASNR method outperforms many other state-of-the-art CS image recovery methods.

\section{APPENDIX A: Proof of Theorem 1}

Owing to the assumption that $\boldsymbol{e}(\boldsymbol{j})$ follows an independent zero mean distribution with variance $\sigma^{2}$, namely, $\boldsymbol{E}[\boldsymbol{e}(\boldsymbol{j})]$ and $\boldsymbol{V a r}[\boldsymbol{e}(\boldsymbol{j})]=\sigma^{2}$. Thus, it can be deduced that each $e(j)^{2}$ is also independent, and the meaning of each $\boldsymbol{e}(\boldsymbol{j})^{2}$ is:

$$
\boldsymbol{E}\left[\boldsymbol{e}(\boldsymbol{j})^{2}\right]=\boldsymbol{V a r}[\boldsymbol{e}(\boldsymbol{j})]+[\boldsymbol{E}[\boldsymbol{e}(\boldsymbol{j})]]^{2}=\sigma^{2}, j=1,2, \ldots, N
$$

By invoking the law of Large numbers in probability theory, for any $\epsilon>0$, it leads to $\lim _{N \rightarrow \infty} \boldsymbol{P}\left\{\left|\frac{1}{N} \sum_{j=1}^{N} \boldsymbol{e}(\boldsymbol{j})^{2}-\sigma^{2}\right|<\frac{\epsilon}{2}\right\}=1$, namely,

$$
\lim _{N \rightarrow \infty} \boldsymbol{P}\left\{\left|\frac{1}{N}\|\boldsymbol{x}-\boldsymbol{r}\|_{2}^{2}-\sigma^{2}\right|<\frac{\epsilon}{2}\right\}=1
$$

Next, we denote the concatenation of all the patches $\boldsymbol{x}_{i}$ and $\boldsymbol{r}_{i}, i=$ $1,2, \ldots, n$, by $\boldsymbol{x}_{l}$ and $\boldsymbol{r}_{l}$, respectively. Meanwhile, we denote the error of each element of $\boldsymbol{x}_{l}-\boldsymbol{r}_{l}$ by $\boldsymbol{e}_{l}(k), k=1,2, \ldots, K$. We have also denote $\boldsymbol{e}_{l}(k)$ following an independent zero mean distribution with variance $\sigma^{2}$.

Therefore, the same process applied to $\boldsymbol{e}_{l}(k)^{2}$ yields $\lim _{N \rightarrow \infty} \boldsymbol{P}\left\{\mid \frac{1}{N} \Sigma_{k=1}^{N} \boldsymbol{e}_{l}(k)^{2}-\right.$ $\left.\sigma^{2} \mid<\frac{\epsilon}{2}\right\}=1$, i.e.,

$$
\lim _{N \rightarrow \infty} \boldsymbol{P}\left\{\left|\frac{1}{N} \sum_{i=1}^{n}\right|\left|\boldsymbol{x}_{l}-\boldsymbol{r}_{l} \|_{2}^{2}-\sigma^{2}\right|<\frac{\epsilon}{2}\right\}=1
$$

Obviously, considering Eqs.(30) and (31) together, we can prove Eq.(19). 


\section{Acknowledgment}

This work was supported by the Natural Science Foundation of China (61462052, 61571220) and the open research fund of National Mobile Commune. Research Lab., Southeast University (No.2015D08).

\section{References}

1. Emmanuel J Candès et al. Compressive sampling. In Proceedings of the international congress of mathematicians, volume 3, pages 1433-1452. Madrid, Spain, 2006.

2. David L Donoho. Compressed sensing. Information Theory, IEEE Transactions on, 52(4):1289-1306, 2006

3. ME Gehm, R John, DJ Brady, RM Willett, and TJ Schulz. Single-shot compressive spectral imaging with a dual-disperser architecture. Optics Express, 15(21):14013-14027, 2007.

4. Marco F Duarte, Mark A Davenport, Dharmpal Takhar, Jason N Laska, Ting Sun, Kevin E Kelly, Richard G Baraniuk, et al. Single-pixel imaging via compressive sampling. IEEE Signal Processing Magazine, 25(2):83, 2008.

5. Yasunobu Hitomi, Jinwei Gu, Mohit Gupta, Tomoo Mitsunaga, and Shree K Nayar. Video from a single coded exposure photograph using a learned over-complete dictionary. In Computer Vision (ICCV), 2011 IEEE International Conference on, pages 287-294. IEEE, 2011.

6. Emmanuel J Candes and Terence Tao. Decoding by linear programming. Information Theory, IEEE Transactions on, 51(12):4203-4215, 2005.

7. Mário AT Figueiredo, Robert D Nowak, and Stephen J Wright. Gradient projection for sparse reconstruction: Application to compressed sensing and other inverse problems. Selected Topics in Signal Processing, IEEE Journal of, 1(4):586-597, 2007.

8. Joel Tropp, Anna C Gilbert, et al. Signal recovery from random measurements via orthogonal matching pursuit. Information Theory, IEEE Transactions on, 53(12):46554666, 2007.

9. Ingrid Daubechies, Michel Defrise, and Christine De Mol. An iterative thresholding algorithm for linear inverse problems with a sparsity constraint. arXiv preprint math/0307152, 2003

10. Andrei Nikolaevich Tikhonov and VB Glasko. Use of the regularization method in non-linear problems. USSR Computational Mathematics and Mathematical Physics, 5(3):93-107, 1965.

11. David Mumford and Jayant Shah. Optimal approximations by piecewise smooth functions and associated variational problems. Communications on pure and applied mathematics, 42(5):577-685, 1989.

12. Tony Chan, Selim Esedoglu, Frederick Park, and A Yip. Recent developments in total variation image restoration. Mathematical Models of Computer Vision, 17, 2005.

13. Antoni Buades, Bartomeu Coll, and Jean-Michel Morel. A non-local algorithm for image denoising. In Computer Vision and Pattern Recognition, 2005. CVPR 2005. IEEE Computer Society Conference on, volume 2, pages 60-65. IEEE, 2005.

14. Weisheng Dong, Lei Zhang, Guangming Shi, and Xiaolin Wu. Image deblurring and super-resolution by adaptive sparse domain selection and adaptive regularization. Image Processing, IEEE Transactions on, 20(7):1838-1857, 2011.

15. Gabriel Peyré. Image processing with nonlocal spectral bases. Multiscale Modeling $\&$ Simulation, 7(2):703-730, 2008.

16. Qian Liu, Caiming Zhang, Qiang Guo, Hui Xu, and Yuanfeng Zhou. Adaptive sparse coding on pca dictionary for image denoising. The Visual Computer, pages 1-15, 2015.

17. Guy Gilboa and Stanley Osher. Nonlocal operators with applications to image processing. Multiscale Modeling \& Simulation, 7(3):1005-1028, 2008.

18. Miyoun Jung, Xavier Bresson, Tony F Chan, Luminita Vese, et al. Nonlocal mumfordshah regularizers for color image restoration. Image Processing, IEEE Transactions on, 20(6):1583-1598, 2011. 
19. Weisheng Dong, Lei Zhang, Guangming Shi, and Xin Li. Nonlocally centralized sparse representation for image restoration. Image Processing, IEEE Transactions on, 22(4):1620-1630, 2013.

20. Michal Aharon, Michael Elad, and Alfred Bruckstein. A k-svd: An algorithm for designing overcomplete dictionaries for sparse representation. Signal Processing, IEEE Transactions on, 54(11):4311-4322, 2006.

21. Michael Elad and Michal Aharon. Image denoising via sparse and redundant representations over learned dictionaries. Image Processing, IEEE Transactions on, 15(12):3736$3745,2006$.

22. Adit Bhardwaj and Shanmuganathan Raman. Robust pca-based solution to image composition using augmented lagrange multiplier (alm). The Visual Computer, pages $1-10,2015$.

23. Ubiratã A Ignácio and Cláudio R Jung. Block-based image inpainting in the wavelet domain. The Visual Computer, 23(9-11):733-741, 2007.

24. Michael Elad and Irad Yavneh. A plurality of sparse representations is better than the sparsest one alone. Information Theory, IEEE Transactions on, 55(10):4701-4714, 2009.

25. Matan Protter, Irad Yavneh, and Michael Elad. Closed-form mmse estimation for signal denoising under sparse representation modeling over a unitary dictionary. Signal Processing, IEEE Transactions on, 58(7):3471-3484, 2010.

26. Xiaolin Wu, Xiangjun Zhang, and Jia Wang. Model-guided adaptive recovery of compressive sensing. In Data Compression Conference, 2009. DCC'09., pages 123-132. IEEE, 2009.

27. Saiprasad Ravishankar and Yoram Bresler. Mr image reconstruction from highly undersampled k-space data by dictionary learning. Medical Imaging, IEEE Transactions on, 30(5):1028-1041, 2011.

28. Lihan He and Lawrence Carin. Exploiting structure in wavelet-based bayesian compressive sensing. Signal Processing, IEEE Transactions on, 57(9):3488-3497, 2009.

29. Lihan He, Haojun Chen, and Lawrence Carin. Tree-structured compressive sensing with variational bayesian analysis. Signal Processing Letters, IEEE, 17(3):233-236, 2010.

30. Jianbo Yang, Xuejun Liao, Xin Yuan, Patrick Llull, David J Brady, Guillermo Sapiro, and Lawrence Carin. Compressive sensing by learning a gaussian mixture model from measurements. Image Processing, IEEE Transactions on, 24(1):106-119, 2015.

31. Sungkwang Mun and James E Fowler. Block compressed sensing of images using directional transforms. In Image Processing (ICIP), 2009 16th IEEE International Conference on, pages 3021-3024. IEEE, 2009.

32. Emmanuel J Candès, Justin Romberg, and Terence Tao. Robust uncertainty principles: Exact signal reconstruction from highly incomplete frequency information. Information Theory, IEEE Transactions on, 52(2):489-509, 2006.

33. Tom Goldstein and Stanley Osher. The split bregman method for 11-regularized problems. SIAM Journal on Imaging Sciences, 2(2):323-343, 2009.

34. Xiaoqun Zhang, Martin Burger, Xavier Bresson, and Stanley Osher. Bregmanized nonlocal regularization for deconvolution and sparse reconstruction. SIAM Journal on Imaging Sciences, 3(3):253-276, 2010.

35. Zhouchen Lin, Minming Chen, and Yi Ma. The augmented lagrange multiplier method for exact recovery of corrupted low-rank matrices. arXiv preprint arXiv:1009.5055, 2010.

36. Jian Zhang, Debin Zhao, and Wen Gao. Group-based sparse representation for image restoration. Image Processing, IEEE Transactions on, 23(8):3336-3351, 2014.

37. Li Xu, Cewu Lu, Yi Xu, and Jiaya Jia. Image smoothing via 10 gradient minimization. In ACM Transactions on Graphics (TOG), volume 30, page 174. ACM, 2011.

38. Karnran Sharifi and Alberto Leon-Garcia. Estimation of shape parameter for generalized gaussian distributions in subband decompositions of video. Circuits and Systems for Video Technology, IEEE Transactions on, 5(1):52-56, 1995.

39. Weisheng Dong, Guangming Shi, Xin Li, Lei Zhang, and Xiaolin Wu. Image reconstruction with locally adaptive sparsity and nonlocal robust regularization. Signal Processing: Image Communication, 27(10):1109-1122, 2012. 
40. Julien Mairal, Francis Bach, Jean Ponce, and Guillermo Sapiro. Online learning for matrix factorization and sparse coding. The Journal of Machine Learning Research, 11:19-60, 2010.

41. Shenlong Wang, Lei Zhang, Yan Liang, and Quan Pan. Semi-coupled dictionary learning with applications to image super-resolution and photo-sketch synthesis. In Computer Vision and Pattern Recognition (CVPR), 2012 IEEE Conference on, pages 2216-2223. IEEE, 2012.

42. Renjie Huang, Mao Ye, Pei Xu, Tao Li, and Yumin Dou. Learning to pool high-level features for face representation. The Visual Computer, 31(12):1683-1695, 2015.

43. Jin Zhan, Zhuo Su, Hefeng $\mathrm{Wu}$, and Xiaonan Luo. Robust tracking via discriminative sparse feature selection. The Visual Computer, 31(5):575-588, 2015.

44. Sungkwang Mun and James E Fowler. Residual reconstruction for block-based compressed sensing of video. In Data Compression Conference (DCC), 2011, pages 183-192. IEEE, 2011.

45. Stephen Becker, Jérôme Bobin, and Emmanuel J Candès. Nesta: a fast and accurate first-order method for sparse recovery. SIAM Journal on Imaging Sciences, 4(1):1-39, 2011.

46. Jian Zhang, Debin Zhao, Chen Zhao, Ruiqin Xiong, Siwei Ma, and Wen Gao. Image compressive sensing recovery via collaborative sparsity. Emerging and Selected Topics in Circuits and Systems, IEEE Journal on, 2(3):380-391, 2012.

47. Karen Egiazarian, Alessandro Foi, and Vladimir Katkovnik. Compressed sensing image reconstruction via recursive spatially adaptive filtering. In Image Processing, $200 \%$. ICIP 2007. IEEE International Conference on, volume 1, pages I-549. IEEE, 2007.

48. Jian Zhang, Chen Zhao, Debin Zhao, and Wen Gao. Image compressive sensing recovery using adaptively learned sparsifying basis via 10 minimization. Signal Processing, 103:114-126, 2014.

49. Thuong Nguyen Canh, Khanh Quoc Dinh, and Byeungwoo Jeon. Multi-scale/multiresolution kronecker compressive imaging. In Image Processing (ICIP), 2015 IEEE International Conference on, pages 2700-2704. IEEE, 2015.

50. Amir Beck and Marc Teboulle. Fast gradient-based algorithms for constrained total variation image denoising and deblurring problems. Image Processing, IEEE Transactions on, 18(11):2419-2434, 2009. 